University of Rhode Island

DigitalCommons@URI

Civil \& Environmental Engineering Faculty

Publications

Civil \& Environmental Engineering

2022

\title{
Predicting the Near Field Underwater Explosion Response of Coated Composite Cylinders using Multiscale Simulations, Experiments, and Machine Learning
}

\author{
Sumeru Nayak \\ University of Rhode Island \\ Gideon A. Lyngdoh \\ University of Rhode Island \\ Arun Shukla \\ University of Rhode Island, shuklaa@uri.edu \\ Sumanta Das
Follow this and additional, works at: https://digitalcommons.uri.edu/cve_facpubs \\ The University of Rhode Island Faculty have made this article openly available. \\ Please let us know how Open Access to this research benefits you.
}

This is a pre-publication author manuscript of the final, published article.

Terms of Use

This article is made available under the terms and conditions applicable towards Open Access

Policy Articles, as set forth in our Terms of Use.

\section{Citation/Publisher Attribution}

Nayak, S., Lyngdoh, G. A., Shukla, A., \& Das, S. (2022). Predicting the Near Field Underwater Explosion Response of Coated Composite Cylinders using Multiscale Simulations, Experiments, and Machine Learning. Composite Structures, 283, 115157. https://doi.org/10.1016/j.compstruct.2021.115157 Available at: https://doi.org/10.1016/j.compstruct.2021.115157

This Article is brought to you for free and open access by the Civil \& Environmental Engineering at DigitalCommons@URI. It has been accepted for inclusion in Civil \& Environmental Engineering Faculty Publications by an authorized administrator of DigitalCommons@URI. For more information, please contact digitalcommonsgroup@uri.edu. 


\title{
Predicting the Near Field Underwater Explosion Response of Coated Composite Cylinders using Multiscale Simulations, Experiments, and Machine Learning
}

\author{
Sumeru Nayak ${ }^{1}$, Gideon A. Lyngdoh ${ }^{1}$, Arun Shukla ${ }^{2}$, and Sumanta Das ${ }^{3 *}$ \\ ${ }^{1}$ Graduate Student, Civil and Environmental Engineering, University of Rhode Island, Kingston, RI, \\ USA \\ ${ }^{2}$ Simon Ostrach Professor, Mechanical, Industrial and Systems Engineering, University of Rhode Island, \\ Kingston, RI, USA \\ ${ }^{3 *}$ Assistant Professor, Civil and Environmental Engineering, University of Rhode Island, Kingston, RI \\ 02881, USA, Email: sumanta_das@ uri.edu (corresponding author)
}

\begin{abstract}
Prediction of underwater explosion response of coated composite cylinders using machine learning (ML) requires a large, consistent, accurate, and representative dataset. However, such reliable large experimental dataset is not readily available. Besides, the ML algorithms need to abide by the fundamental laws of physics to avoid non-physical predictions. To address these challenges, this paper synergistically integrates $M L$ with high-throughput multiscale finite element $(F E)$ simulations to predict the response of coated composite cylinders subjected to nearfield underwater explosion. The simulated responses from the multiscale approach correlate very well with the experimental observations. After validation of the multiscale approach, a representative and consistent dataset containing more than 3800 combinations is developed using high-throughput multiscale simulation by varying the fiber/matrix/coating material properties, coating thickness as well as experimental variables such as explosive energy and stand-off distance. The dataset is leveraged to predict the response of coated composite cylinders subjected to nearfield underwater explosion using a feed-forward multilayer perceptron-based neural network (NN) approach which shows excellent predictions. Overall, the synergistic approach powered by physics-based simulations presented here can potentially enable materials scientists and engineers to make intelligent, informed decisions in the purview of innovative design strategies for underwater explosion mitigation in composite structures.
\end{abstract}

Keywords: Composite materials; Underwater explosion; Blast loading; Finite Element; Multiscale simulations; Machine Learning 


\section{INTRODUCTION}

Throughout the twenty-first century, the use of polymer composites in marine structures has grown rapidly owing to their high specific stiffness, strength, superior corrosion resistance, low infrared, magnetic, and radar cross-sectional signatures when compared to conventional metal alloys. As the naval vessels are designed to sustain high-intensity blast waves toward mission capability [1], it becomes imperative to study the high strain rate response of such composite structures. The techniques for blast mitigation toward absorption, disruption, and reduction of blast waves can be broadly categorized into increased impedance mismatch (resulting from stress wave reflection at the interface of protective materials and structures) [24], sacrificial claddings (impulse transformation for reducing stress transfer and increased duration of load application) [5-8], geometrical arrangements (resulting in redirecting blast waves away from structure) $[9,10]$ and blast wave disrupters brought about by barriers between blast wave and structure $[11,12]$. In the purview of the maritime defense industry, polymer composite structures with impedance mismatch (for instance sandwich composites) and claddings have been shown to mitigate blast loads efficiently [13-15]. The performance of these composite structures is commonly evaluated in terms of the out-of-plane and inplane behavior, particularly the displacements and particle velocity which can quantify the energy dissipation. In order to evaluate blast mitigation performance of composite structures subjected to underwater explosion (UNDEX) events, fluid-structure interaction (FSI) studies form an integral part. In a bid to increase blast mitigation efficiency, elastomeric coatings over composite structures have garnered significant attention in recent years $[8,16]$. The rate-sensitive behavior of such polymers introduces nonlinear response and dispersive wave propagation in the structures.

While understanding the fundamental composition-property relationships at several interactive length scales are extremely crucial toward the efficient design of high-performance underwater composite structures for enhanced explosion mitigation, complex hierarchical characteristics of laminated polymer composites and its minute structural/behavioral intricacies at multiple hierarchical length scales makes it exceedingly challenging to elucidate such relationships experimentally. A few studies have performed numerical simulations toward the prediction of the near field underwater explosion response of composite structures [8,16-18]. While these studies are focused on macro-scale structural response evaluation, significant strides have to be made to develop holistic approaches linking hierarchical material behavior at multiple interactive length scales to macro-scale structural responses to enable comprehensive tuning of composite structures at various targeted hierarchical length scales for efficient underwater explosion mitigation. Toward this end, numerical simulations integrating multiple interactive length scales to the macro scale can serve as an efficient tool. However, multiscale finite element simulation models with regard to predicting the near field underwater explosion response of composites are computationally expensive 
because of hierarchical intricacies at multiple length scales. To alleviate such computational burden, computationally efficient and user-friendly Machine Learning (ML)-based predictive tools can be implemented. Such ML-based predictive models can be used as a starting point toward enabling the development of simple and user-friendly materials design tools that can be easily leveraged by designers and decision-makers for widespread application of these emerging coated composite materials against underwater explosion event. However, design of composite materials and structures for efficient underwater explosion mitigation using ML presents various well-established challenges. First, ML approaches would require an accurate, consistent, and representative large dataset. Such large experimental data for near field underwater explosion response of composite structures are limited given the complexity in laboratory or real-scale testing. Second, owing to the black-box nature of ML techniques such as neural network [19], its indiscriminate use can lead to non-physical solutions [20,21].

To overcome these limitations, this paper forwards a viable strategy through synergistic integration of highthroughput multiscale numerical simulations with ML for prediction of underwater explosion response of coated composite cylinders. Owing to the intricate nature of near-field loadings that lead to localized damage in the composites, this study implements a multi-scale rate-dependent predictive approach that accounts for the inherent heterogeneity of the structure at multiple hierarchical length scales. This highthroughput multiscale simulation approach is leveraged to obtain a large dataset of composition-dependent underwater explosion responses of composite cylinders. Moreover, the response obtained from multiscale numerical simulation approach are also validated with experimental observations. Hence, such high throughput multiscale simulation-based data generation follows the fundamental laws of physics and helps to circumvent various challenges related to obtaining non-physical solutions. Neural network (NN) is chosen here in this paper primarily because of its potential high accuracy of prediction as demonstrated by several materials-design studies [22,23]. Although NN yields high prediction-accuracy, its interpretability has been challenging and its ability to provide new physical insights has been limited [21,24]. Toward this end, in this paper, we have adopted a recent approach called SHapley Additive exPlanations (SHAP) $[25,26]$ to interpret the results obtained from NN. Overall, the synergistically integrated high-throughput multiscale FE simulation and ML-based approach presented here has a high potential of generating new knowledge which will enable materials scientists and engineers to make intelligent, informed decisions regarding the selection of compositions as well as features of underwater composite structures for efficient explosion mitigation and thus accelerate their acceptance and utilization.

\section{EXPERIMENTAL RESPONSE EVALUATION}

This section briefly describes the UNDEX experiments which involve a large tank (see Figure 1) hosting the explosive event and high-speed cameras for the evaluation of the structural response using digital image 
correlation (DIC). Pressure sensors and DIC enable quantification of the transient response of the cylinders. The experimental setup and techniques have been adequately detailed in the authors' previous publications $[8,16]$. The objective of carrying out the experiments is to have the predictive model correlated with the experimental observations for an RP-503 charge placed at a standoff distance of $5.08 \mathrm{~cm}$ from a polyurea coated cylinder. The cylinder configuration (see Figure 1) has a wall thickness of $1.14 \mathrm{~mm}$, length $40.6 \mathrm{~cm}$, and outside diameter of $7.44 \mathrm{~cm}$ while the coating thickness is $1.9 \mathrm{~mm}$. A brief description of the experimental method is provided hereafter.

\subsection{Experimental Method}

The experiments for the explosive event are carried out in a large diameter $(2.1 \mathrm{~m})$ water-filled cylindrical pressure vessel with an array of windows facilitating viewing and recording of experiments with cameras. The laminated cylinder has a laminate schedule of 0 and 45 plies stacked in a 0/45/45/0 configuration.

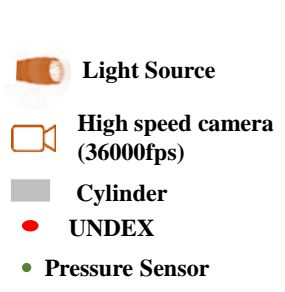

- Pressure Sensor

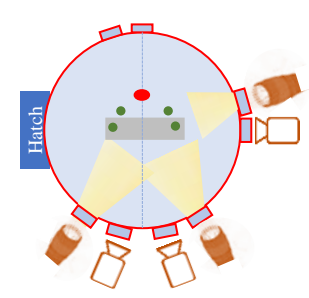

(a)

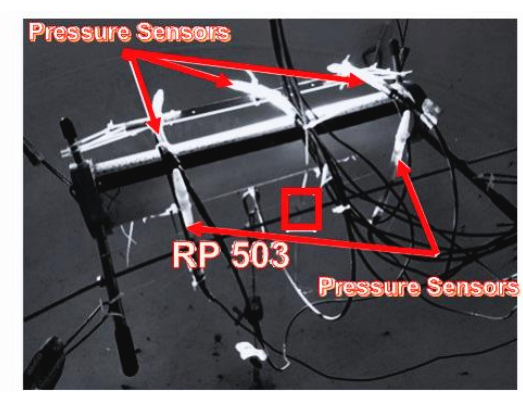

(b)

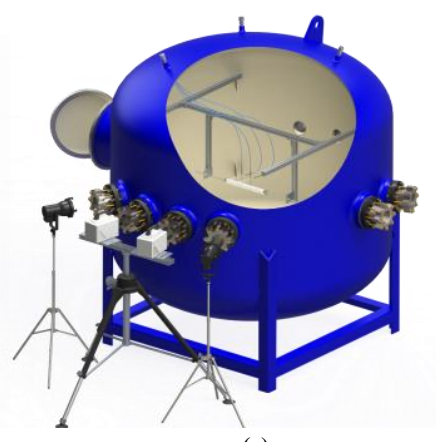

(c)

Figure 1. (a) Schematic representation of the experimental setup (b) Support wire-cage with pressure sensors and charge (c) 3D view of the experimental tank

The cylinder is suspended with tensioning cables that restrict rigid body translation during loading. A combination of three high-speed video recorders (each capturing frames at $36000 \mathrm{fps}$ ) FastCam SA1 was used to capture the profile and a stereoscopic view of the event under illuminated conditions. Random speckles on white painted cylinders facilitate DIC on the cylinders on post-processing of the captured frames using the VIC-3D software [27]. The pressure sensors used are PCB 148A05 tourmaline dynamic sensors recording at a sampling rate of $2 \mathrm{MHz}$. A wire support cage secures the pressure sensors and explosive (see Figure 1(b)). It is to be noted that the cylinders are sealed against water ingress by ensuring sealed contact with the endcaps. Figure 1 (c) shows the 3D view of the experimental setup with the cylinder suspended inside the tank. 


\subsection{Experimental Results}

The experiments carried out on water-submerged cylinders are analyzed hereafter for the pressure and DICenabled displacement histories. These observations enable validation of the simulation framework as described later in Section 3.2.3. The pressure sensors, as shown in Figure 1 are mounted on the near charge face and the far-face. The pressure-time histories of the charge are shown in Figure 2 on the charge-side and far-side of the cylinder. It is to be noted that the pressure-time history shown in Figure 2 corresponds to the initial stage before cavitation sets in since the evaluation of displacements using DIC was possible till that stage. Hence the pressure-time history shown here does not represent the reflected pulses and bubble collapse as excessive displacements during those stages make it challenging to obtain meaningful correlations between frames so as to obtain full-field displacement using DIC. The center-line displacement of the near-field of the cylinder is shown in Figure 3 (a) for coated and uncoated configurations at $1 \mathrm{~ms}$. Figures 3 (b) and (c) show the displacement contours at $2.25 \mathrm{~ms}$ for a standoff distance of $2.54 \mathrm{~cm}$ and 5.08 $\mathrm{cm}$ respectively.

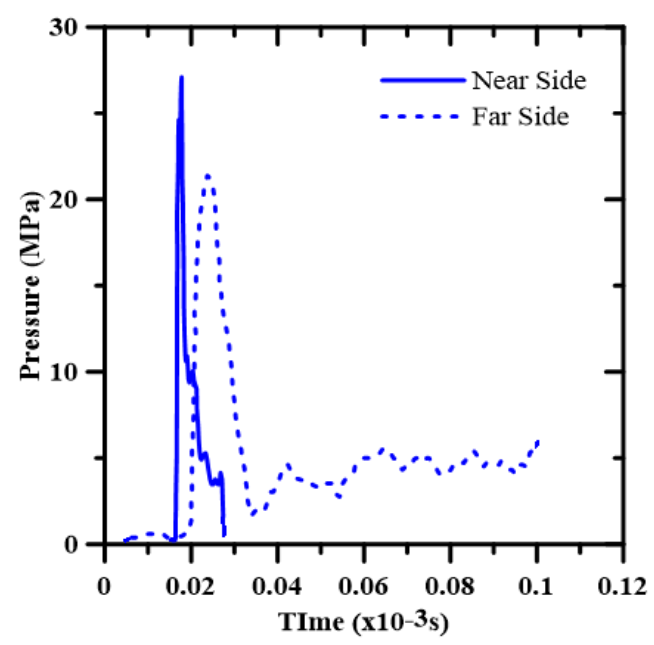

Figure 2. Pressure-time histories on near-face and far-face of the cylinder for a representative RP-503 charge placed at a standoff distance of $5.08 \mathrm{~cm}$ 

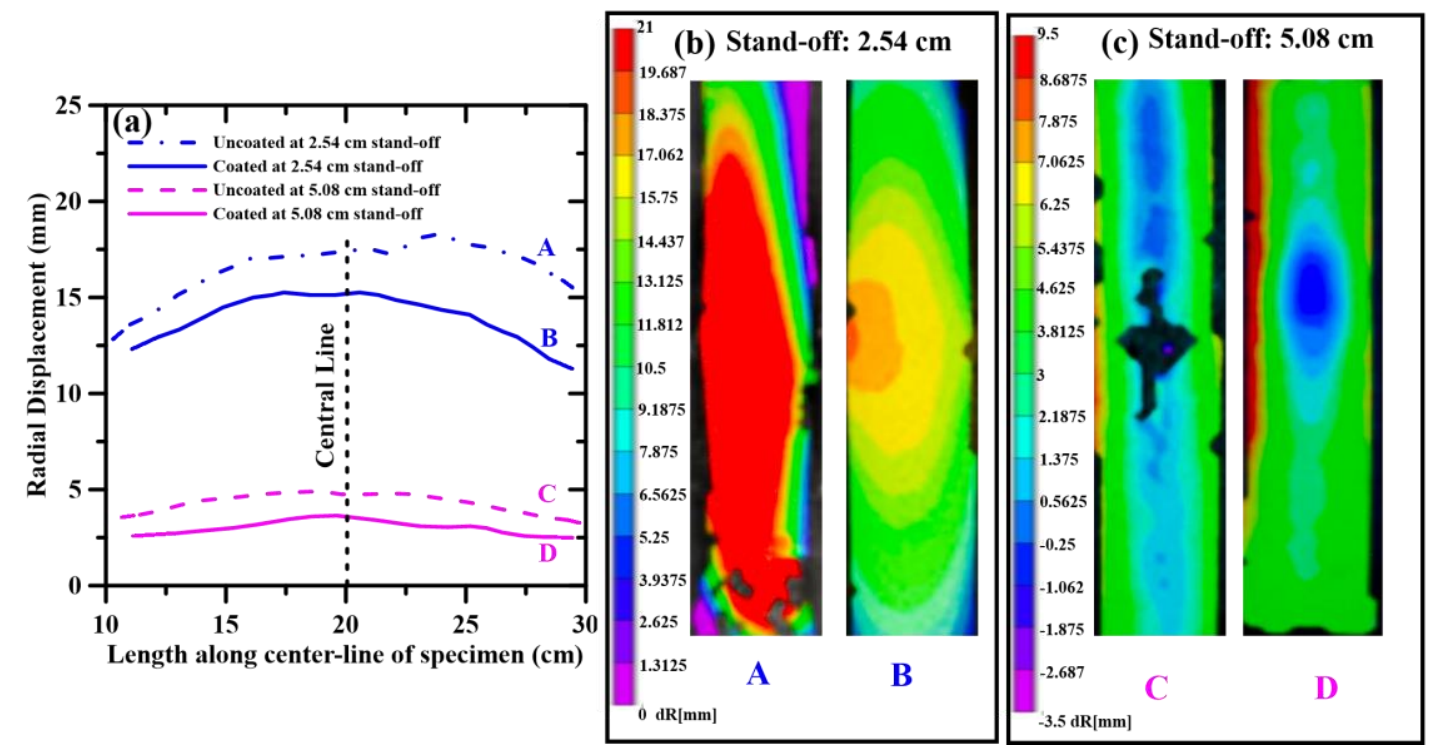

Figure 3. Radial Displacements: (a) Along the central length for coated and uncoated configurations at 1ms; Radial Displacement Contours at 2.25ms for a stand-off distance of (b) $2.54 \mathrm{~cm}$ and (c) $5.08 \mathrm{~cm}$. A and C show uncoated configurations whereas B and D represent coated cylinders.

The radial displacement of the center lines on the cylinder configurations (uncoated for A and C; coated for B and D (see Figure 3)) are observed for the loading events till DIC was possible as explained earlier. As observed from the displacement contours, for both standoff distances $(2.54 \mathrm{~cm}$ and $5.08 \mathrm{~cm})$, the coated configurations show a significantly lower displacement as compared to the uncoated cylinder. This proves the effectiveness of the coating in mitigating the effects of near field explosion. The experimental observations obtained here are compared later with predicted responses from multiscale numerical simulations for verification and validation of the numerical models.

\section{MULTISCALE NUMERICAL SIMULATION TO OBTAIN LARGE DATASET}

\subsection{Multiscale Simulation Approach}

Prediction of near field underwater explosion response of coated composite cylinders requires ratedependent mechanical properties of the constituent laminates and the coating. While the stiffness and strength for the constituents can be obtained experimentally under static or dynamic conditions, the choice of multi-scale analysis provides a reliable and flexible method for strength and stiffness prediction by accounting for the distinct characteristics of the constituents under various loading scenarios under high strain rates. Thus, the implementation of multiscale approach helps improve the robustness of the overall predictive framework by reducing the dependence on the experimental inputs. Moreover, multiscale approach is integrated with the machine learning strategy by facilitating large dataset generation. The 
forthcoming subsections detail the hierarchical multiscale material structure as well as the simulation approach.

\subsubsection{Material structure at multiple length scales}

To obtain a large dataset for the dynamic performance of laminated composite cylinders subjected to nearfield underwater explosion, this paper implements a multiscale numerical simulation strategy that facilitates information exchange between the macroscale laminated cylinder, the mesoscale woven unit cell, and the micro-scale hexagonal fiber-matrix unit cell. The dataset, generated using a multiscale numerical simulation approach, is used later for ML-based performance prediction of composite cylinders subjected to nearfield explosion. A brief description of the adopted interactive length scales for response evaluation is presented here in Figure 4.

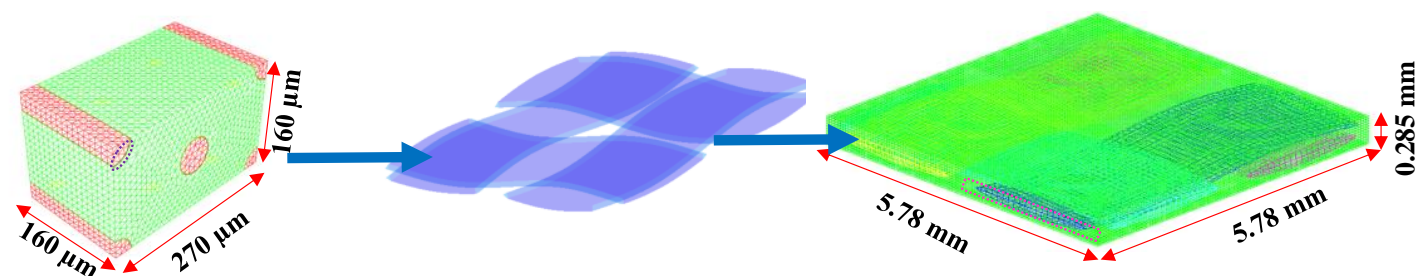

(a)

(b)
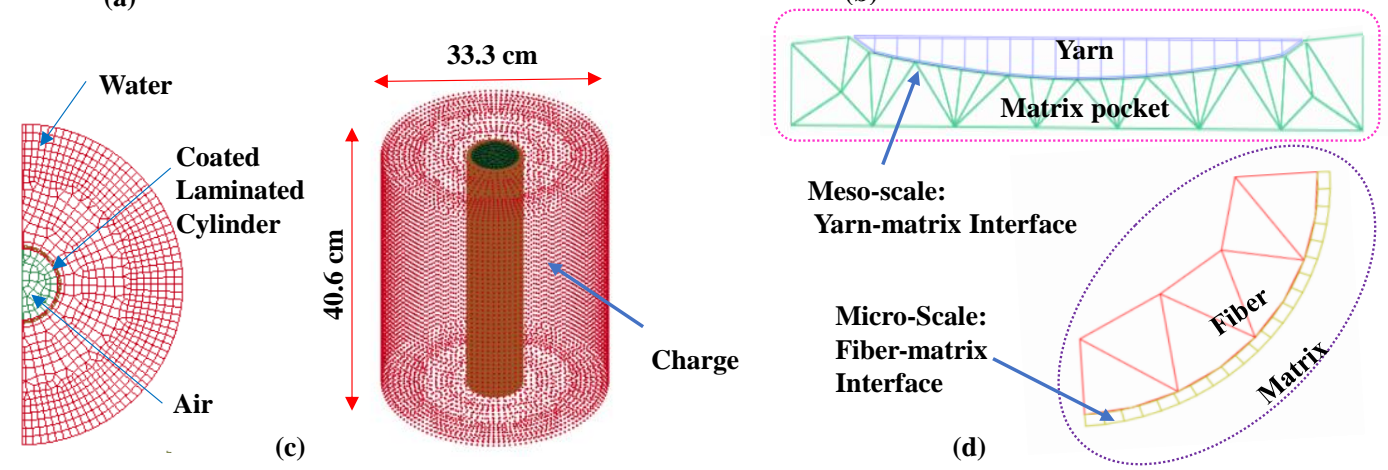

Figure 4. Material structure at multiple interactive length scales: (a) RVE for yarns at the microscale: threephase hexagonal yarn unit cell containing fibers embedded in the matrix and the fiber-matrix interface elements (b) Yarn cross-over within the plain weave unit cell at mesoscale and mesoscale plain weave unit cell containing the yarns and the matrix; (c) Sectional top view of the macro-scale composite cylinder model with multi-material groups and full macro-scale model configuration for FSI UNDEX simulation for an explosive charge at a standoff distance equaling $5.08 \mathrm{~cm}$. It is to be noted that the radial distance of the fluid domain boundary from the cylinder surface is $~ 2.54$ times the standoff distance for simulating nonreflecting boundary conditions, and (d) enlarged view of interface nodal connections at yarn-matrix (mesoscale) interface and fiber-matrix interface (microscale).

The multiscale analysis starts at the micro-scale unit cell (Figure 4(a)) of yarns containing fiber inclusions, matrix, and the fiber-matrix interface elements. The homogenized moduli and strength obtained from the micro-scale analysis are used as inputs for the yarn behavior in the mesoscale where the yarns are embedded in matrix pockets with cross-overs representing a $2 \times 2$ plain weave (Figure 4(b)). The yarns are treated as 
unidirectional laminates with yarn-yarn and yarn-matrix interfaces. It is to be noted that since $2 \times 2$ plain woven fabrics are considered in the study, the ply behavior is transversely isotropic although the framework is capable of modeling unbalanced fabrics. The composite rate-dependent moduli and strengths obtained from the mesoscale simulations pertaining to tension, compression, and shear are used as inputs for the simulation of the dynamic response of macroscale composite cylinder (Figure 4(c)) subjected to nearfield underwater explosion. In particular, the rate-dependent homogenized behavior from the mesoscale serves as input to the homogenized shell representing a ply. The plies are stacked in defined orientations and the interlaminar characteristics are implemented. Finally, a solid coating with rate-dependent characteristics is implemented at the exposed surface of the cylinder. The configuration is thereafter modeled in a surrounding fluid domain with an explosive charge and subjected to a near-field explosion. The nodal connectivity at meso- (yarn-matrix surface interaction) and micro-scale (fiber matrix cohesive elements) are shown in Figure 4(d). The parallax view of the terminal nodes of the yarn and the matrix appears to be non-adjacent in Figure 4(d) because of the curved nature of the terminal surface of the yarns. Although this paper implements coated cylindrical geometry for the model, the simulation approach can handle any type of geometries designed based on various functional requirements. The following sub-sections elaborate the numerical approaches at each length scale in the multi-scale framework.

\subsubsection{Numerical approach at micro-scale}

The salient features for a micro-mechanical analysis are provided hereafter.

\subsubsection{Geometry Generation and Boundary Conditions}

The representative hexagonal unit cell $(160 \mu \mathrm{m} \times 160 \mu \mathrm{m} \times 270 \mu \mathrm{m})$ of the periodic fiber arrays within the tows of the weave, is shown in Figure 4 (a). Any further increase in the size of the unit cell resulted in an insignificant change in the obtained responses. The diameter of glass fiber is $20 \mu m$ [28]. Periodic boundary conditions (PBCs) [29,30] are implemented for uniaxial (tension/compression) and shear loadings. PBC implementation process is adequately detailed in the authors' previous publications [29,30]. A brief description of the PBC application is mentioned herewith for ease of reference. Periodic hex-dominated mesh is generated on the geometry and the resulting nodes are divided into sets of face nodes, edge nodes, and corner nodes for application of suitable constraints as described in [31]. For any pair of nodes $A$ and $B$ lying on parallel boundary surfaces of the unit cells, their relative displacements $\left(u_{i}\right)$ along $x, y$ and $z$ directions denoted by $i=x, y, z$ are shown in Equation 1 [32].

$$
u_{i}^{A}-u_{i}^{B}=\epsilon_{a v g} \Delta x_{k}
$$


where $\epsilon_{\text {avg }}$ is the average strain in the unit cell and $\Delta x_{k}$ is the position vector connecting the nodes $A$ and $B$ lying on parallel boundary surfaces. Under such conditions, traction and displacement continuities are maintained for parallel surfaces, as demonstrated in [33]. Periodic boundary conditions serve as an effective tool for property prediction in systems with embedded inclusions. For such systems with multiple planes of symmetry, the efficiency of periodic boundary conditions are described in [34].

\subsubsection{Rate dependent plasticity of matrix}

The constitutive behavior of polymer matrices should account for the rate-dependent inelastic behavior and the load cases (tension/compression/shear). The total strain $\left(\varepsilon_{i j}^{T}\right)$ is composed of elastic $\left(\varepsilon_{i j}^{E}\right)$ and inelastic $\left(\varepsilon_{i j}^{I}\right)$ strains. The total stress $\left(\sigma_{i j}^{T}\right)$ is divided into hydrostatic $\left(\sigma_{k k}^{h y d}\right)$ and deviatoric $\left(\sigma_{i j}^{d e v}\right)$ components. Hereafter, $J_{2}$ denotes the second invariant of the deviatoric stress tensor $\sigma_{i j}^{d e v}$. The formulations of the material behavior are characterized by the inelastic strain rate tensor $\dot{\varepsilon}_{i j}^{I}$ in terms of material constants $D_{0}$ and $n$; state variables $Z$ and $\alpha$ as per Equation $2[35,36]$.

$$
\dot{\varepsilon}_{i j}^{I}=2 D_{0} \exp \left[(-0.5)\left(\frac{Z}{\sigma_{e}}\right)^{2 n}\right]\left(\frac{\sigma_{i j}^{d e v}}{2 \sqrt{J_{2}}}+\alpha \delta_{i j}\right)
$$

Where effective stress $\sigma_{e}=\sqrt{3}\left(\sqrt{J_{2}}+\alpha \sigma_{k k}^{h y d}\right)$ and $\delta_{i j}$ is the Kronecker delta. The material constant $D_{0}$ represents the maximum inelastic strain rate while $n$ controls the rate dependency. The state variables $Z$ and $\alpha$ correspond to the internal stress states and hydrostatic stress effects respectively. The deviatoric component of the inelastic strain rate $\dot{\varepsilon}_{i j}^{I}$ is denoted by $\dot{e}_{i j}^{I}$ and defined as per Equation 3.

$$
\dot{e}_{i j}^{I}=\dot{\varepsilon}_{i j}^{I}-\frac{\dot{\varepsilon}_{k k}^{I}}{3} \delta_{i j}
$$

Where $\dot{\varepsilon}_{k k}^{I}$ is the sum of the diagonal components of the $\dot{\varepsilon}_{i j}^{I}$ tensor or the hydrostatic component.

A material constant $q$ that represents the hardening rate and the effective deviatoric inelastic strain rate $\dot{e}_{e}^{I}$ are used to compute the rate of evolution of the state variables as per Equations 4 and 5.

$$
\begin{aligned}
& \dot{Z}=q\left(Z_{1}-Z\right) \dot{e}_{e}^{I} \\
& \dot{\alpha}=q\left(\alpha_{1}-\alpha\right) \dot{e}_{e}^{I}
\end{aligned}
$$

Where $Z_{1}$ and $\alpha_{1}$ are maximum values of the state variables and effective deviatoric inelastic strain rate $\dot{e}_{e}^{I}=\sqrt{\frac{2}{3} \dot{e}_{i j}^{I} \dot{e}_{i j}^{I}}$. The rate-dependent plastic behavior is implemented in the ABAQUS ${ }^{\mathrm{TM}}$ solver via user- 
defined vectorized subroutine as detailed in [37]. A similar approach for the constitutive behavior of matrix [35] has been successfully implemented for epoxy [36] and vinyl ester [38].

\subsubsection{Cohesive interface with frictional slip}

This section explains the behavior of the microscale fiber-matrix interface implemented using cohesive elements. The cohesive zone model (CZM) is used to bring about interface degradation resulting in redistribution of stresses. Progressive debonding of the fiber-matrix interface helps to capture the stress gradients observed at the interfaces. A bilinear traction-separation law is followed to characterize the cohesive elements at the interface of the fiber and the matrix. Before the onset of damage, the linear traction separation law enforces an elastic behavior, as represented by Equation 6.

$$
\left(\begin{array}{l}
t_{n} \\
t_{s} \\
t_{t}
\end{array}\right)=\left(\begin{array}{lll}
K_{n} & & \\
& K_{s} & \\
& & K_{t}
\end{array}\right)\left(\begin{array}{l}
\delta_{n} \\
\delta_{s} \\
\delta_{t}
\end{array}\right)
$$

Where $t_{i}$ are the tractions, $K_{i}$ are stiffnesses and $\delta_{i}$ are separations of contact surfaces $(i=$ $n$ for normal and $s, t$ for shear). For numerical convergence, a large value of stiffness is adopted to represent perfect bonding at the interface elements without separation, as demonstrated in [39]. The damage initiation criterion is shown in Equation 7 [40].

$$
\left(\frac{\left\langle t_{n}\right\rangle}{t_{n}^{0}}\right)^{2}+\left(\frac{\left\langle t_{s}\right\rangle}{t_{s}^{0}}\right)^{2}+\left(\frac{\left\langle t_{t}\right\rangle}{t_{t}^{0}}\right)^{2}=1
$$

Where $\langle x\rangle \in R$ is defined as $(x+|x|) / 2$.

After damage initiation, the progressive damage follows a BK criterion [32] toward softening behavior characterization as shown in Equation 8.

$$
G_{C}=G_{n C}+\left(G_{s C}-G_{n C}\right)\left(\frac{G_{s}+G_{t}}{G_{s}+G_{t}+G_{n}}\right)^{\eta}
$$

Where $G_{C}$ is mixed-mode fracture toughness, $G_{n C}$ and $G_{s C}$ are normal and shear critical toughness of the interface, $\eta$ is a material constant (adopted as 1.45 [40]). The computation of $G_{i}$ (for $i=n, s, t$ ) comprises the area under the corresponding traction-separation curve (for normal and shear loads).

The interface damage variable is defined as follows.

$$
D=\frac{\delta_{m}^{f}\left(\delta_{m}^{\max }-\delta_{m}^{0}\right)}{\delta_{m}^{\max }\left(\delta_{m}^{f}-\delta_{m}^{0}\right)} ; \delta_{m}^{0} \leq \delta_{m}^{\max } \leq \delta_{m}^{f}
$$


Where $\delta_{m}^{\max }$ is the maximum value of $\delta_{m}$ (effective separation) throughout the loading history. The effective separation is given by $\left(\delta_{m}=\sqrt{\left\langle\delta_{n}\right\rangle^{2}+\delta_{s}^{2}+\delta_{t}^{2}}\right)$. The effective separation at the onset of damage denoted by $\delta_{m}^{0}=\left(T_{i n t} / K\right)$ is given by the ratio of interfacial strength $\left(T_{i n t}\right)$ and stiffness $(K)$. The mixedmode separation at failure denoted by $\delta_{m}^{f}$ is given by $2 G_{C} / K \delta_{m}^{0}$ where $G_{C}$ is computed by the B-K equation (see Equation 8). The tractions are continuously updated during the loading process as per the following Equation [40].

$$
\begin{gathered}
t=K \delta \text { for elastic stage }\left(\delta_{m}^{\max }<\delta_{m}^{0}\right) \\
t=(1-D) \bar{t} \text { for softening stage }\left(\delta_{m}^{0}<\delta_{m}^{\max }<\delta_{m}^{f}\right) .
\end{gathered}
$$

Where $\bar{t}$ is the traction stress component calculated by linear-elastic traction separation behavior for the separation displacement.

Equation 10 represents the undamaged part of the interface stresses whereas Equation 11 pertains to damage evolution after onset of fiber-matrix debonding. However, under specific load cases such as transverse compression, a friction law is incorporated in the CZM so as to capture the effect of friction between the fiber and the matrix. Thereafter, a traction-separation term [41] attributed to frictional sliding on the damaged interface $\left(\bar{t}^{d}\right)$ is added to Equation 11 that leads to Equation 12. It is to be noted that only the shear inelastic frictional sliding $\delta_{s}^{d i}$ and $\delta_{t}^{d i}$ contribute to the traction-separation since the normal displacement $\delta_{n}$ doesn't affect the inelastic friction sliding [41,42].

$$
t=(1-D) \bar{t}+\bar{t}^{d}
$$

Here $\bar{t}^{d}=D\left[\begin{array}{ccc}K_{n} & & \\ & K_{s} & \\ & & K_{t}\end{array}\right]\left[\begin{array}{c}-\left\langle-\delta_{n}\right\rangle \\ \delta_{s}-\delta_{s}^{d i} \\ \delta_{t}-\delta_{t}^{d i}\end{array}\right]$ and the evolution of inelastic frictional sliding $\delta_{s, t}^{d i}$ is governed by Equation $13[42,43]$.

$$
\dot{\delta}_{s}^{d i}=\dot{\lambda} \frac{\bar{t}_{s}^{d}}{\sqrt{\left(\bar{t}_{s}^{d}\right)^{2}+\left(\bar{t}_{t}^{d}\right)^{2}}} ; \dot{\delta}_{t}^{d i}=\dot{\lambda} \frac{\bar{t}_{t}^{d}}{\sqrt{\left(\bar{t}_{s}^{d}\right)^{2}+\left(\bar{t}_{t}^{d}\right)^{2}}}
$$

Where $\lambda$ is the effective mixed mode displacement given by $\left(\lambda=\frac{K_{n}\left\langle\delta_{n}\right\rangle^{2}+K_{S} \delta_{S}^{2}+K_{t} \delta_{t}^{2}}{\sqrt{K_{n}\left\langle\delta_{n}\right\rangle^{2}+K_{S} \delta_{S}^{2}+K_{t} \delta_{t}^{2}}}\right)$ and $\bar{t}_{s, t}^{d}$ are the shear components of $\bar{t}^{d}$. The interfacial behavior is implemented in the ABAQUS ${ }^{\mathrm{TM}}$ solver by a userdefined vectorized subroutine [44]. 


\subsubsection{Rate dependence and damage}

The rate-dependent behavior of the matrix is captured by its modulus correlated with the current effective strain rate $\dot{\varepsilon}_{c u r r}^{e f f}$ and the effective reference strain rate $\dot{\varepsilon}_{0}^{\text {eff }}$ as per Equation 14 [45].

$$
E_{m, \dot{\varepsilon}}=E_{m, 0}\left(1+C_{m} \ln \frac{\dot{\varepsilon}_{c u r r}^{e f f}}{\dot{\varepsilon}_{0}^{e f f}}\right)
$$

Where $C_{m}$ is a material constant, $E_{m, \dot{\varepsilon}}$ and $E_{m, 0}$ are moduli at $\dot{\varepsilon}_{c u r r}^{e f f}$ and $\dot{\varepsilon}_{0}^{e f f}$ respectively. While the reference $\dot{\varepsilon}_{0}^{\text {eff }}=1 s^{-1}$ [46] for quasi-static condition, $\dot{\varepsilon}_{\text {curr }}^{\text {eff }}$ is defined as per Equation 15 in terms of the rates of component strain rates $\left(\dot{\varepsilon}_{11}, \dot{\varepsilon}_{22}, \dot{\varepsilon}_{33}, \dot{\varepsilon}_{12}, \dot{\varepsilon}_{23}, \dot{\varepsilon}_{13}\right)$ and mean strain rate $\dot{\varepsilon}_{m}=1 / 3\left(\dot{\varepsilon}_{11}+\dot{\varepsilon}_{22}+\right.$ $\left.\dot{\varepsilon}_{33}\right)$.

$$
\dot{\varepsilon}_{c u r r}^{e f f}=\sqrt{\frac{2}{3}\left[\left(\dot{\varepsilon}_{11}-\dot{\varepsilon}_{m}\right)^{2}+\left(\dot{\varepsilon}_{22}-\dot{\varepsilon}_{m}\right)^{2}+\left(\dot{\varepsilon}_{33}-\dot{\varepsilon}_{m}\right)^{2}+2 \dot{\varepsilon}_{12}^{2}+2 \dot{\varepsilon}_{23}^{2}+2 \dot{\varepsilon}_{13}^{2}\right]}
$$

The strength of the matrix at $\dot{\varepsilon}_{\text {curr }}^{\text {eff }}$ is defined as per Equation 16 [47].

$$
X_{m, \varepsilon}=\left(1+b\left[\dot{\varepsilon}_{c u r r}^{e f f}\right]^{n}\right) X_{m, 0}
$$

Where $b$ and $n$ are material constants, $X_{m, \dot{\varepsilon}}$ corresponds to $\dot{\varepsilon}_{c u r r}^{\text {eff }}$ and $X_{m, 0}$ is quasi-static strength.

Owing to the distinct tensile and compressive behavior of the matrix, a modified von Mises failure criterion is adopted in terms of the tensile and compressive strengths $X_{m}^{T}$ and $X_{m}^{C}$ respectively as described in Equation 17 [48].

$$
\frac{\sigma_{V M}^{2}}{X_{m}^{T} X_{m}^{C}}+\left(\frac{1}{X_{m}^{T}}-\frac{1}{X_{m}^{C}}\right) I_{1}=1
$$

Where $\sigma_{V M}$ is the Von Mises stress and $I_{1}$ is the first stress invariant.

Once the failure criteria is met, an isotropic damage model is initiated [37,45] that progressively degrades the material $\left[\boldsymbol{C}^{\text {dam }}=(1-D) \boldsymbol{C}^{\text {stif }}\right]$ where $\boldsymbol{C}^{\text {stif }}$ and $\boldsymbol{C}^{\text {dam }}$ are undamaged and damaged stiffness respectively and the damage variable, $D$ is defined as per Equation 18.

$$
D=1-\exp \left[1-\frac{\left(\frac{\varepsilon}{\varepsilon_{f}}\right)^{m}}{m}\right]
$$

Where $\varepsilon_{f}$ and $\varepsilon$ are the strains at failure and current states respectively, $m$ is a material parameter. The isotropic damage is implemented in the ABAQUS ${ }^{\mathrm{TM}}$ solver by a user-defined vectorized subroutine [45]. 
The damage model for the matrix is appended with the rate-dependent plastic behavior described earlier in Section 3.1.2.2.

The rate dependence of the fiber behavior is similarly captured by Equation 16 in terms of the strength [47]. The maximum principal stress failure criterion is adopted for the elastic-brittle fibers, as applied to ratedependent simulations [49]. Since multiple user-defined subroutines are used in the model, the subroutines are called by a material name identifier and executed at the material domains. The rate-dependent constitutive behavior obtained from the micro-mechanical analysis is used as an input for the yarns in the mesoscale analysis.

\subsubsection{Numerical approach at mesoscale}

The mesoscale analysis involves a plain weave unit cell with yarns (which are themselves UD laminates) and matrix pockets. The salient features of the analysis are presented hereafter.

\subsubsection{Geometry Generation and Boundary Conditions}

The analysis is performed using a representative $2 \times 2$ plain weave unit cell (see Figure 4(b)) with dimensions of $5.78 \mathrm{~mm} \times 5.78 \mathrm{~mm} \times 0.285 \mathrm{~mm}$. This size of unit cell is adopted here based on a unit cell sensitivity study that shows insignificant changes in the results for bigger unit cells. Here, an elliptical cross-section of yarns (dimensions along the major axis and minor axis being $2.50 \mathrm{~mm}$ and $0.36 \mathrm{~mm}$ respectively) is considered here. A similar approach has been adopted in a simulation study [50] for a glass-fiber reinforced plain weave. Periodic boundary conditions are implemented by mapping periodic meshes on opposite faces. While the $\mathrm{z}$-faces have matrix nodes, the $\mathrm{x}$ and $\mathrm{y}$ faces have both matrix and tow nodes. A node matching algorithm is implemented to obtain a conformal mesh (see Figure 4(d)) on those faces thus facilitating the implementation of periodic boundary conditions. The PBC formulations are adequately detailed in a previous publication by the authors [30]. A Python implementation in TexGen software [51,52] is used to obtain the conformal mesh for the yarns owing to its superiority over voxel meshes [53] for interface definitions.

\subsubsection{Contact behavior at the yarn-matrix and yarn-yarn interfaces}

The mesoscale unit cell has matrix pockets surrounding the yarns while the yarns cross-over each other leading to yarn-yarn and yarn-matrix contact pairs. The interfacial behavior is defined by the cohesive surface with the yarn surface as the master surface and the yarn/matrix surface as slave surface for the yarn- 
yarn and yarn-matrix contact pairs respectively. The definitions for the cohesive contact property include normal behavior, cohesive behavior, and damage [54]. Normal behavior determines the contact behavior in the normal direction and is controlled by the hard contact pressure overclosure relationship. The cohesive behavior enables the contact surfaces to be controlled by a traction-separation behavior. The slave nodes are released from the master surface when the interface stress meets the cohesive contact failure criterion. After release, the interpenetration between the contact pair is prevented by contact. The failed cohesive surfaces have a friction defined in the formulations. A bilinear traction-separation law is modeled in the interfaces which have been demonstrated successfully for fiber-matrix interfacial behavior $[55,56]$. The formulations adopted here are the same as the ones adopted at the microscale for the fiber-matrix interfaces as defined in Equations 6-12. Such a technique has been successfully implemented for impact analyses [57] and woven unit cells [53].

\subsubsection{Failure Analysis}

The anisotropic behavior of the tows is captured in three directions - along fiber direction (in-plane fill), transverse direction (in-plane warp), and out-of-plane direction, denoted by a, b and c respectively [58]. The elastic moduli (tensile $E_{a}^{T}, E_{b}^{T}, E_{c}^{T}$ and compressive $\left.E_{a}^{C}, E_{b}^{C}, E_{c}^{C}\right)$; shear moduli $\left(G_{a b}, G_{b c}, G_{c a}\right.$ along $a-$ $b, b-c$ and a-c planes); axial strengths (tensile $X_{a}^{T}, X_{b}^{T}$ and compressive $\left.X_{a}^{C}, X_{b}^{C}\right)$; shear strength $\left(S_{a b}\right.$ along in-plane) characterize the stiffness matrix and the failure criteria. Here, the Chang-Chang failure criteria [58] is implemented for simulating progressive damage in the yarn of the mesoscale weave. Successful implementation of Chang-Chang failure criteria has been demonstrated in [59]. Multiple failure modes govern the damage initiation in the fiber and matrix followed by a material degradation simulated by deletion of failed elements. As the tow is itself a matrix-fiber composite, the failure modes are distinct for fibers (tensile/compressive failure modes denoted by $e_{f}$ and $e_{c}$ respectively as shown in Equations 19-20) and matrix (tensile/compressive failure modes denoted by $e_{m}$ and $e_{d}$ respectively as shown in Equations 21-22). It is to be noted that the in-plane shear failure criterion for matrix is given by $\left[\left(\frac{\sigma_{a b}}{s_{c}}\right)^{2}-1\right]>0$ which if true, initiates matrix damage.

$$
\begin{aligned}
& e_{f}^{2}=\left(\frac{\sigma_{a a}}{X_{a}^{T}}\right)^{2}+\beta\left(\frac{\sigma_{a b}}{S_{c}}\right)-1(\text { tensile fiber mode }) \\
& e_{c}^{2}=\left(\frac{\sigma_{a a}}{X_{a}^{C}}\right)^{2}-1(\text { compressive fiber mode }) \\
& e_{m}^{2}=\left(\frac{\sigma_{b b}}{X_{b}^{T}}\right)^{2}+\left(\frac{\sigma_{a b}}{S_{c}}\right)^{2}-1(\text { tensile matrix mode })
\end{aligned}
$$




$$
e_{d}^{2}=\left(\frac{\sigma_{b b}}{2 S_{c}}\right)^{2}+\left[\left(\frac{X_{a}^{C}}{2 S_{c}}\right)^{2}-1\right] \frac{\sigma_{b b}}{X_{b}^{C}}+\left(\frac{\sigma_{a b}}{S_{c}}\right)^{2}-1(\text { compressive matrix mode })
$$

The failure criteria in Equations 19 and 21 are checked when $\sigma_{a a}>0$ or $\sigma_{b b}>0$ (for tensile cases). The failure criteria in Equations 20 and 22 are checked when $\sigma_{a a}<0$ or $\sigma_{b b}<0$ (for compressive cases). Owing to the anisotropic behavior of the tows, the modes in Equations 19-22 are checked in the orthogonal direction as well. The material parameter $\beta$ (see Equation 19) is adopted as 0, as suggested in [58] after experimental validation. The failure criteria are implemented in a vectorized user subroutine for ABAQUS $^{\mathrm{TM}}[60]$.

\subsubsection{Rate dependence}

The correlation between the strength and strain rate (see Equation 16), as obtained from the homogenized hexagonal unit cells in the micro-scale are used as input to the mesoscale yarns for governing the failure criteria in Equations 19-22. For the matrix pockets, a similar rate-dependent model is adopted, as detailed in Section 3.1.2.4.

\subsubsection{Numerical approach at the macro-scale}

The lamina behavior obtained from the mesoscale is implemented as ply-behavior in the laminated cylinder subjected to nearfield underwater explosion. The forthcoming sub-sections describe the numerical simulation methodology for the macro scale fluid-structure interaction (FSI) problem.

\subsubsection{ALE Technique and methodology}

In the case of FSI events subjected to nearfield underwater explosion, the fluid mesh experiences large deformations making the application of purely Lagrangian methods computationally expensive and inefficient. Purely Lagrangian algorithms adopt individual nodes of the computational mesh that follows the associated particle during motion and are used in structural mechanics. Although it enables easy tracking of free surfaces and material interfaces with history-dependent constitutive relations, its weakness lies in its inability to follow large distortions of the computational domain without recourse to frequent remeshing operations.

On the other hand, the purely Eulerian approach is typically used for fluids and it is not suitable for structural deformation since the mesh is fixed and not allowed to deform. Eulerian algorithms are widely used in fluid dynamics where the computational mesh is fixed and the continuum moves with respect to the grid. In the Eulerian description, large distortions in the continuum motion can be handled with relative ease, but generally at the expense of precise interface definition and resolution of flow details. For efficient 
computations, this paper adopts Arbitrary Lagrangian-Eulerian (ALE) method [17] that combines the Lagrangian and Eulerian techniques. Because of the shortcomings of purely Lagrangian and purely Eulerian descriptions, the Arbitrary Lagrangian-Eulerian or ALE technique [17,61] combines the best features of both. In ALE, the nodes of the computational mesh may be moved with continuum in normal Lagrangian fashion or be held fixed in an Eulerian manner or be moved in some arbitrarily specified way to give a continuous rezoning capability. Because of this freedom in moving the computational mesh offered by ALE description, greater distortions of the continuum can be handled than would be allowed by a purely Eulerian approach. Additionally, the ALE description can accommodate significant distortions of the computational mesh while preserving the clear delineation of interfaces typical of a purely Lagrangian approach. The governing equations in ALE are the conservation equations for energy, mass, and momentum which are conserved and advected from one element to another. Conservation equations are used to solve fluid mesh velocities and displacements. The conservation of mass, momentum, and energy are given in Equations 2325.

$$
\begin{gathered}
\frac{\partial \rho}{\partial t}=(-\rho) \operatorname{div}(v)-\left(v_{i}-u_{i}\right) \frac{\partial \rho}{\partial x_{i}} \\
\rho \frac{\partial v_{j}}{\partial t}=\sigma_{i, j}-\rho\left(v_{i}-u_{i}\right) \frac{\partial v_{j}}{\partial x_{j}} \\
\rho \frac{\partial e}{\partial t}=\sigma_{i j} \varepsilon_{i j}-\rho\left(v_{i}-u_{i}\right) \frac{\partial e}{\partial x_{j}}
\end{gathered}
$$

Where $\rho$ and $x$ are mass density and displacement respectively; $v$ and $u$ are the fluid velocity and the mesh velocity respectively and $e$ is the energy. The Lagrangian formulation for the conservation Equations 2325 sets the mesh velocity equal to the particle velocity $(u=v)$ and calculates the solution for deformed mesh. On the other hand, the Eulerian formulations for the conservation equations set the mesh velocity $u=0$ resulting in a fixed mesh. For ALE, in modern hydrocodes like LS-DYNA, a mesh rezoning algorithm (smoothing algorithms) is implemented wherein the element nodes are rezoned to an optimal position thus preventing excessive distortion in the fluid mesh. An advection step follows the rezoning that transports material flux from one element to the other. The amount of material flux is also determined by the rezoning algorithm. This rezoning technique significantly reduces computation time. In contrast, purely Lagrangian techniques don't use rezoning while the purely Eulerian approach rezones the element nodes to their original position.

\subsubsection{Macro-scale Multi-material Constitutive Behavior}

The Jones-Wilkins-Lee (JWL) equation of state (EOS) [17,58], shown in Equation 26 is adopted for explosion analysis by using an exponential function form. 


$$
p=A\left(1-\frac{\omega}{R_{1} V}\right) e^{-R_{1} V}+B\left(1-\frac{\omega}{R_{2} V}\right) e^{-R_{2} V}+\frac{\omega E}{V}
$$

Where $A, B, R_{1}, R_{2}$ and $\omega$ are material constants; $E$ is the internal energy per unit volume and $V$ is the initial relative volume. Equation 26 enables the computation of relationships between relative density, pressure, and relative volume. Standard relationships between relative volume and pressure for different explosives, available in the literature [17], are adopted here.

For water, Gruneisen EOS [58] is used to calculate its internal characteristics. Such an implementation facilitates the implementation of shockwave propagation and the resulting tension/compression in fluids. The shockwave propagation in an underwater explosion event is captured by incorporating a non-linear shock velocity-particle velocity relationship. The Gruneisen EOS with a cubic shock velocity $\left(u_{s}\right)$, particle velocity $\left(u_{p}\right)$ defines pressure for compressed material as per Equation 27 [58].

$$
p=\frac{\rho_{0} C^{2} \mu\left[1+\left(1-\gamma_{0} / 2\right) \mu-\frac{a}{2} \mu^{2}\right]}{\left[1-\left(S_{1}-1\right) \mu-S_{2}\left(\mu^{2} / \mu+1\right)-S_{3}\left(\mu^{3} /(\mu+1)^{2}\right)\right]^{2}}+\left(\gamma_{0}+a \mu\right) E
$$

where $E$ is the internal energy per unit volume, $\rho_{0}$ is the initial density, $C$ is the intercept of $u_{s}-u_{p}$ curve, $\gamma_{0}$ is the Gruneisen gamma, $a$ is a first-order volume correction to $\gamma_{0}$. The material constants are $C, S_{1}, S_{2}$ and $S_{3}$. The parameter $\mu$ is directly related to the relative volume $V\left(\mu=\frac{1}{V}-1\right)$. For expanded materials, the pressure is defined by Equation 28 [58].

$$
p=\rho_{0} C^{2} \mu+\left(\gamma_{0}+a \mu\right) E
$$

For a Linear Polynomial EOS [58], the pressure is given by Equation 29.

$$
p=C_{0}+C_{1} \mu+C_{2} \mu^{2}+C_{3} \mu^{3}+\left(C_{4}+C_{5} \mu+C_{6} \mu^{2}\right) E
$$

Where $E$ is the internal energy per unit volume, $\mu=\frac{\rho}{\rho_{0}}-1$ for $\rho$ is current density and $\rho_{0}$ is reference density. For a gas with gamma law equation of state such as air, $C_{0}=C_{1}=C_{2}=C_{3}=C_{6}=0$ and $C_{4}=$

$C_{5}=\gamma-1$ where $\gamma=\frac{C_{p}}{C_{v}}$ (ratio of specific heats at constant pressure and constant volume) [58]. Thereafter, the pressure for a perfect gas is given by Equation 30 [58].

$$
p=(\gamma-1) \frac{\rho}{\rho_{0}} E
$$

\subsubsection{Underwater explosion and shockwave}

The four primary characteristics associated with a shockwave by an explosion include peak pressure, pressure-time history, shockwave impulse, and shockwave energy flux density. The explosive constants 
used in the following formulations ( $K_{1}$ and $A_{1}$ for peak pressure in Equation 31, $K_{2}$ and $A_{2}$ for shockwave time in Equation 32, $K_{3}$ and $A_{3}$ for shockwave impulse in Equation 33, $K_{4}$ and $A_{4}$ for shockwave energy flux density in Equation 34) are available in literature [17]. The peak pressure is given by Equation 31 [17].

$$
P_{\max }=K_{1}\left(\frac{W^{1 / 3}}{R}\right)^{A_{1}}
$$

Where $R$ is the distance of the point of interest from the center of the charge in feet, $W$ is the charge weight in pounds-force. To determine the pressure-time history of point $R$ for the shockwave, an exponential decay of peak pressure is assumed. The time constant, $\theta$ is the time it takes for the pressure to fall to $36.8 \%$ of the peak pressure, calculated by $\left(\theta=W^{1 / 3} K_{2}\left(\frac{W^{1 / 3}}{R}\right)^{A_{2}}\right)$. The pressure-time history at a point $R$ is given by Equation 32.

$$
P(t)=P_{\max } e^{-t-t_{1} / \theta}
$$

Where $P_{\max }$ is given by Equation 31 and $t_{1}$ is the time the shockwave takes to reach the point of interest $R$ with the speed of sound $C$ given by $\left(t_{1}=R / C\right)$. The shockwave impulse $I$ and energy flux density $E$ are given by Equations 33 and 34 respectively.

$$
\begin{gathered}
I=K_{3} W^{1 / 3}\left(\frac{W^{1 / 3}}{R}\right)^{A_{3}} \\
E=K_{4} W^{1 / 3}\left(\frac{W^{1 / 3}}{R}\right)^{A_{4}}
\end{gathered}
$$

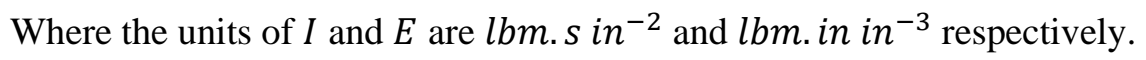

\subsubsection{Model Implementation}

The finite element model representing the dynamic response of composite cylinder subjected to underwater explosion in Figure 4(c) consists of the cylinder body, coating, surrounding water tank, internal air, and the charge. As mentioned in Section 3.1.1, the inclusion of the domain to a distance larger than twice the standoff value ensures the boundary effects do not influence the fluid-structure interaction. Furthermore, the use of such a sub-domain for the modeling of the corresponding experiments is deemed appropriate as the loading of the cylinder and subsequent response occurs sufficiently fast that reflections from the tank do not affect the overall transient response of the cylinder [16]. Thus, a non-reflecting boundary condition (*BOUNDARY_NON_REFLECTING in LS-Dyna) is used to model the outer surface of the fluid subdomain. This allows the associated pressure waves to leave the domain (a situation similar to a free field 
detonation). In other words, the non-reflecting boundary ensures that the pressure waves do not reflect off the free surface.

ALE multi-material element formulations are used in LS-Dyna with Type 11 solid elements for the Eulerian components (for air, water, and the explosive charge). An equation of state (EOS) combined with a material model captures the fluid/explosive components of the model. The details of the EOS definitions are mentioned in Section 3.1.4.2 for air, water and explosives. For both water and air, the *MAT_NULL material is defined in LS-Dyna. For water, the material definition is coupled with Gruneisen EOS, whereas Linear Polynomial EOS is adopted for air. For water, the density and sound speed are $1 \mathrm{~g} / \mathrm{cm}^{3}$ and $149000 \mathrm{~cm} / \mathrm{s}$ respectively. The density of air is $0.0013 \mathrm{~g} / \mathrm{cm}^{3}$. The material model adopted for the explosive charge is *MAT_HIGH_EXPLOSIVE_BURN in LS-Dyna which is combined with the JWL EOS.

The structural aspect of the coupled model consists of the composite cylinder and the coating. The cylinder is free to move within the fluid domain since there were no rigid constraints applied during the experiments. The composite cylinder is modeled with Belytschko-Tsay shell elements (*SECTION_SHELL definition in LS-Dyna) with a layer of shell elements that enables the composite layup with 2 integration points per layer so as to capture the correct bending behavior per ply, as explained in [62]. The ICOMP flag enables orthotropic material behavior for the shell elements. The coating material is represented in the model by solid elements with a constant stress formulation and the coatings are considered to be perfectly bonded to the substrate, as observed from the experiments [16]. *MAT_ENHANCED_COMPOSITE_DAMAGE definition was implemented in LS-Dyna for the composite laminate while *MAT_SIMPLIFIED_RUBBER with rate-dependence was implemented for the coating respectively. The implementation of Chang-Chang failure criteria [47,59] for simulating progressive damage analysis in the fabric in macro-scale has been demonstrated successfully in [47]. In the fabric laminate, the Equations 19-22 (see Section 3.1.3.3) are checked for failure in in-plane fill, in-plane warp directions. The through-thickness delamination behavior of the plies is captured by *AUTOMATIC_SURFACE_TO_SURFACE_TIE_BREAK in LS-Dyna. The contact failure is governed by the interlaminar strength in the normal and tangential directions, denoted by NFLS and SFLS respectively [58], as described in Equation 35.

$$
\left(\frac{\left|\sigma_{n}\right|}{N F L S}\right)^{2}+\left(\frac{\left|\sigma_{S}\right|}{S F L S}\right)^{2} \geq 1
$$

Where $\sigma_{n}$ and $\sigma_{s}$ are the normal and tangential stresses at the ply-ply contact surface. The element deletion governed by the Chang-Chang failure criterion and the interlaminar damage brought about by surface tie breaks facilitate the progressive damage in the laminated fabric composite. A similar approach was 
successfully implemented in a recent publication [47]. The multiscale framework described above is used later for generating datasets on which ML is applied.

\subsection{Multiscale Simulation Results and Correlation with Experimental Observations}

\subsubsection{Micro-scale Response}

In the micro-scale unit cell, the periodic boundary conditions are implemented to apply time-dependent strain in tension, compression for along-fiber and transverse directions; shear for in-plane and out-of-plane directions. For every load case, a stress update algorithm (based on the approach detailed in section 3.1.2 and shown in Figure A.1 in Supplementary material Section A) is implemented in the matrix and interface until fiber damage is initiated. A mesh-convergence study was performed and a mesh containing 331836 (C3D10 for matrix and fiber, COH3D6 for interface in ABAQUS ${ }^{\mathrm{TM}}$ ) elements was chosen for further analysis in the micro-scale (please refer to Section $\mathrm{C}$ of the Supplementary material). The constitutive behavior of the unit cell is detailed in Section 3.1.2. A homogenization scheme is implemented for the effective property prediction [29,30]. The elastic moduli are computed from the linear elastic behavior of the matrix-fiber unit cell prior to interfacial debonding. As the interfacial debonding sets in, the stress concentration in the matrix rises leading to matrix yielding and subsequent failure. The fiber damage is initiated at the fiber strength corresponding to the load case under consideration. At the point of fiber damage initiation, the strength of the yarn is computed.

For microscale analyses with fiber and matrix under various loading directions, the homogenized behavior of the unit cell is determined by its components: fiber, matrix, and interface depending on the loading directions. For along-fiber loading, the homogenized unit cell behavior is dominated by the fiber properties. For transverse and shear loadings, the homogenized unit cell behavior is primarily governed by matrix behavior and interface behavior. The input properties to the matrix (see Section 3.1.2.2) are adopted for epoxy and vinyl ester from [35] and [38] respectively. The quasi-static glass fiber properties are adopted from [30]. The rate-dependent coefficients for moduli and strength (see Equations 14 and 16) for the matrix and fibers are adopted from [47]. The interfacial strengths in the normal and shear directions are adopted as $28 \mathrm{MPa}$ and $40 \mathrm{MPa}$ respectively [63]. The critical energy release rates (see Equation 8) are taken as $0.01 \mathrm{~N} / \mathrm{mm}$ and $0.025 \mathrm{~N} / \mathrm{mm}$ for normal and shear behavior respectively [64]. All the input values are provided in Section B-1 of the Supplementary material.

For the transverse loading cases, special considerations need to be incorporated [41,65-68] for enhancing the prediction capabilities of micromechanical models. For transverse tension and compression, a normal critical energy release rate of $0.002 \mathrm{~N} / \mathrm{mm}$ is used for the interface as adopted from the literature [41,67]. The transverse compression case incorporates frictional behavior $(\mu=0.4)$ [41] in the interface (see 
Equation 13) for enhancing the predictive capability of the micromechanical model [41]. For the transverse tension, a rate-dependent factor $\left(K=\frac{1-V_{f}\left(1-\frac{E_{m, \dot{\varepsilon}}}{E_{f, \dot{\varepsilon}}}\right)}{1-\sqrt{4 V_{f} / \pi}\left(1-\frac{E_{m, \dot{\varepsilon}}}{E_{f, \dot{\varepsilon}}}\right)}\right)$ is used to obtain the UD strength $X^{T}$ with the matrix tensile strength $X_{m}^{T}$ and matrix Poisson's ratio $v_{m}$ as per $\left(X^{T}=\frac{1-v_{m}}{K\left(1+v_{m}\right)\left(1-2 v_{m}\right)} X_{m}^{T}\right)$, as detailed in a previous publication [68]. The factored strength shows a better match with experimental observations $[69,70]$.

Representative damage states of the unit cell under along-fiber, transverse, and shear loading conditions are shown in Figures 5(a-b), Figures 5(c-d), and Figures 5(e-f) respectively.

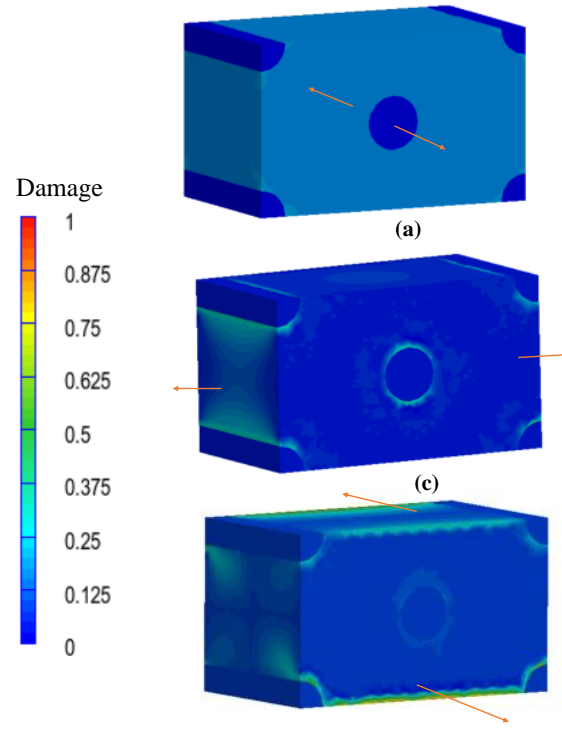

(e)

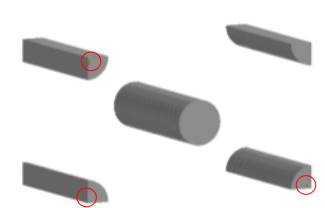

(b)

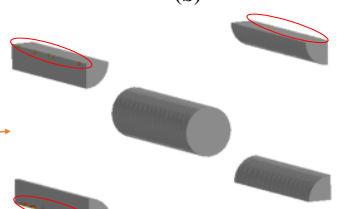

(d)

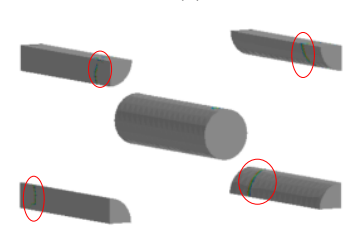

(f)

Figure 5: Damage states in representative micro-scale (fiber-matrix) unit cell (size $160 \mu \mathrm{m}$ x $160 \mu \mathrm{m}$ x $270 \mu \mathrm{m}$ ) subjected to various load cases corresponding to a strain rate of 600/s for Along-fiber loading (ab), Transverse loading (c-d), Shear loading (e-f); Onset of damage for the load cases are shown in (a),(c) and (e) for matrix and (b),(d),(f) for fiber (encircled in red)

While the Figures 5(a),(c) and (e) show the matrix damage onset at a strain of 0.015, 0.012 and 0.001 respectively, Figures 5(b), (d) and (f) exhibit the fiber damage onset at a strain of 0.022, 0.02 and 0.0015 respectively.

The predicted rate-dependent moduli and strengths for the homogenized yarns are shown in Figure 6(a) and (b) respectively for various load combinations. 

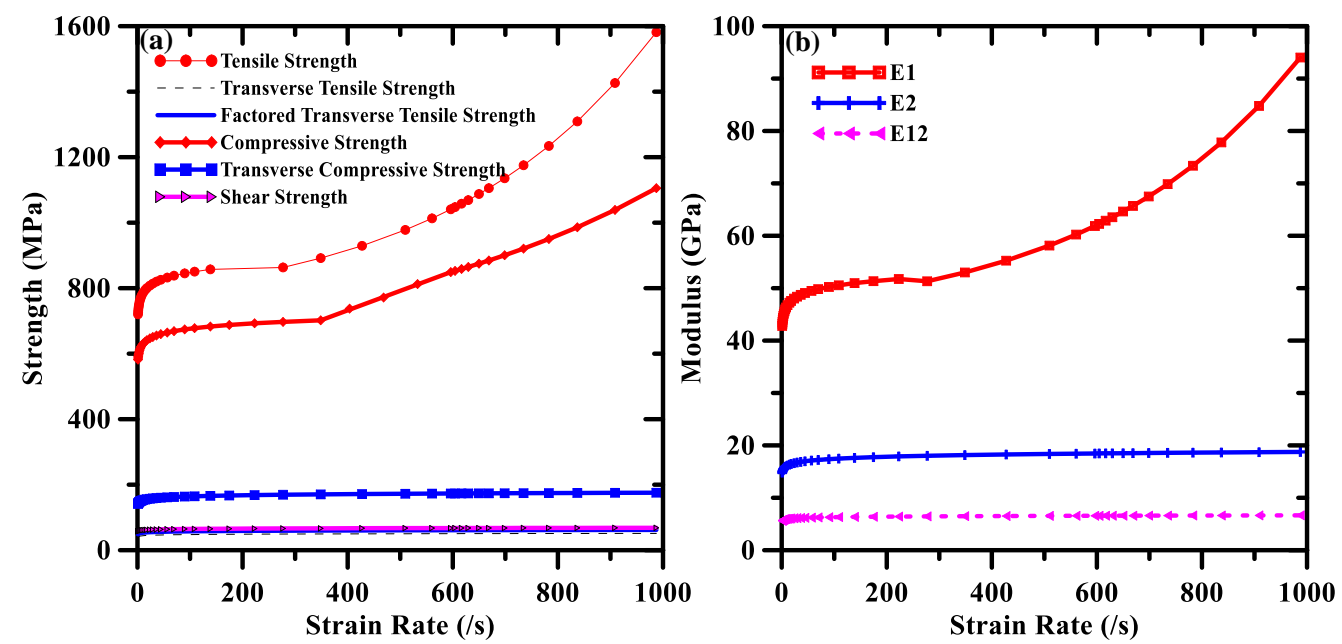

Figure 6. Homogenized rate-dependent micro-scale properties for (a) strength and (b) moduli that serve as input for the mesoscale yarns (E1 denotes along-fiber tensile modulus, E12 denotes in-plane shear modulus and E2 denotes transverse tensile modulus)

For microscale analyses, the rate-dependent fiber and matrix properties serve as input and the homogenized unit cell properties are characterized with rate-dependent moduli and rate-dependent strengths (see Figure 6). These rate-dependent properties serve as input to the tows in the subsequent mesoscale weave unit cell. The rate dependence of the transverse properties is nuanced when compared to along-fiber load cases which can be attributed to the transverse properties being governed primarily by the matrix and interface input properties.

\subsubsection{Meso-scale Response}

In the mesoscale, the behavior of transversely isotropic fabric is ascertained along in-plane fill and in-plane warp directions. For every load case, a stress update algorithm (based on the approach detailed in section 3.1.3 and shown in Figure A.2 in Supplementary material Section A) is implemented in the matrix, yarn, and yarn-matrix/yarn-yarn contacts as per the constitutive behavior detailed in Section 3.1.3. The representative mesh, chosen based on mesh-convergence study, as elaborated in Section $\mathrm{C}$ of the Supplementary material, comprises 2223642 C3D10 elements in ABAQUS ${ }^{\mathrm{TM}}$ (see Figure C.1(b) in the Supplementary material). The interfacial strength is $27.6 \mathrm{MPa}$ and 10.3 MPa for normal and shear behavior [47] respectively whereas the critical energy release rates are $0.280 \mathrm{~J} / \mathrm{mm}^{2}$ and $1.45 \mathrm{~J} / \mathrm{mm}^{2}$ along normal and shear directions [30] respectively. The strength of the fabric is predicted at the point of damage initiation in the yarn [47]. Since the plain weave unit cell represents a balanced fabric, it has the same number of fiber tows in warp and weft directions thus justifying its transversely isotropic behavior. The inputs to the fiber tows are distinct for along-fiber and transverse loadings but the homogenized behavior of the woven unit 
cell is transversely isotropic. While the homogenized micro-scale properties are used as material inputs for the yarns, the matrix pockets adopt similar properties as provided in Section B-1 of the Supplementary material. The input interfacial properties are provided in Section B-2 of the Supplementary material (see Table B.3 in the Supplementary material).

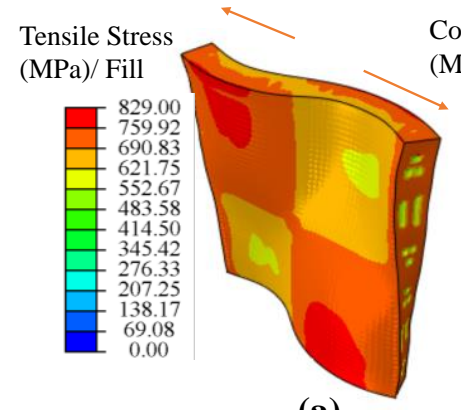

(a)
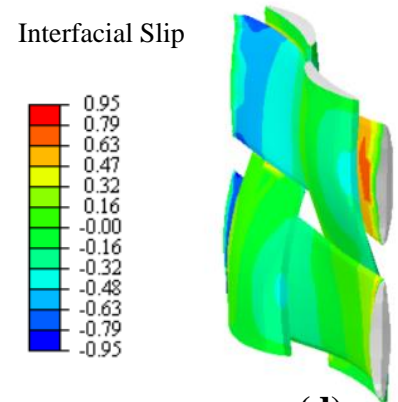

(d)

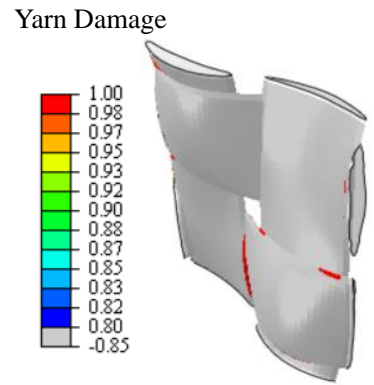

(g)

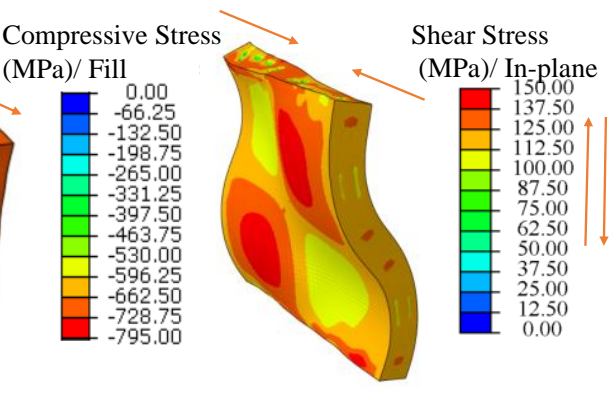

(b)

Interfacial Slip

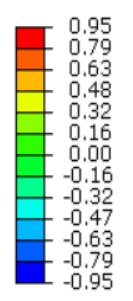

Yarn Damage

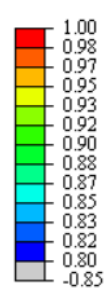

(h)

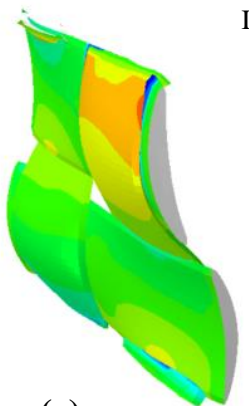

(e)

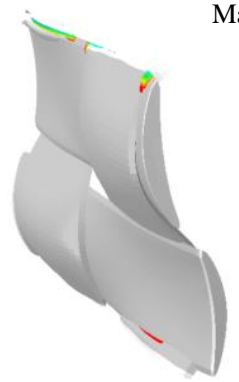

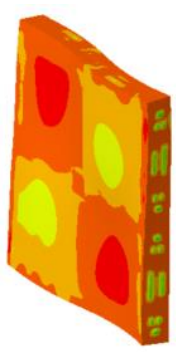

(c)

Interfacial Slip

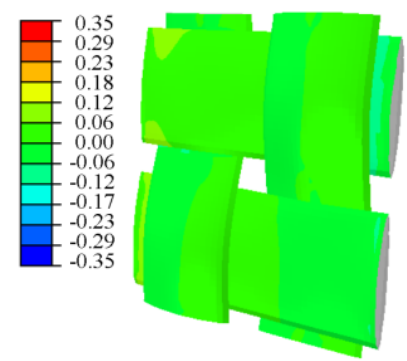

(f)

Matrix Damage

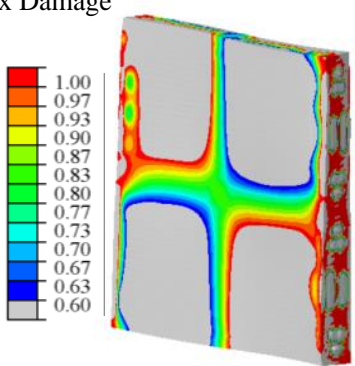

(i)

Figure 7. Contours for deformed $2 \times 2$ plain weave unit cells corresponding to a strain rate of $600 / \mathrm{s}$ for different loading directions ( $\mathrm{a}, \mathrm{d}$ and $\mathrm{g}$ for tension); ( $\mathrm{b}, \mathrm{e}$, and $\mathrm{h}$ for compression); (c, $\mathrm{f}$ and $\mathrm{i}$ for shear); (a), (b) and (c): stress contours for tension, compression, and shear respectively; (d), (e) and (f): interfacial slip contour for tension, compression, and shear respectively; (g), (h) for fiber damage and (i) matrix damage status for tension, compression and shear respectively

Figure 7 shows the representative stress, slip, and damage response (tension, compression, and shear) at the mesoscale under a strain rate of 600/s. Figures 7 (a), (b) and (c) show representative stress contours for tension (applied strain 0.01), compression (applied strain 0.012) and shear conditions (applied strain 0.0025 ) respectively. Figures 7 (d), (e), and (f) demonstrate the slip contours at the yarn-matrix and yarn- 
yarn interfaces due to applied tensile, compressive and shear strains respectively. The figures clearly show equal and opposite slips at the yarn ends, a reflection of the periodic nature of the unit cell. The matrix damage status under tension, compression, and shear are shown in Figures 7 (g), (h), and (i) respectively. It is to be noted that the matrix being significantly weaker shows a higher degree of damage than the yarns (which, in itself is a UD laminate). From the results, it can be inferred that Figures 7 (g-h) and 7 (i) pertain to the dominant failure modes (yarn damage and matrix shear cracking respectively).

The homogenized behavior of the woven unit cell is captured for tension, compression, and in-plane shear. The rate-dependent characteristics of the woven unit cell serve as inputs for lamina properties in the subsequent macro-scale laminated cylinder. This rate-dependence of the lamina behavior is characterized in terms of the strength for loading cases of tension, compression, and shear. However, the moduli input to the macro-scale lamina is considered rate-independent, a strategy consistent with high-rate dynamic simulations [47]. Although rate-dependent moduli are used in thick laminates [71] where through-thickness behavior is a major consideration, the current scope of study implements 4-layered laminates which are significantly thinner. It is to be noted that the multiscale framework presented in the current study can sufficiently capture the through-thickness behavior of the woven unit cell, as elaborated in Supplementary Section D (see Figure D.1) for through-thickness compression.

Owing to the balanced nature of the fabric, the axial moduli are considered as $24.65 \mathrm{GPa}$ while the shear modulus is $4.82 \mathrm{GPa}$, consistent with [72]. The predicted rate-dependent strengths for the homogenized yarns are shown in Figure 8 for tension, compression, and shear. The current scope of application implements a built-in material model in LS-DYNA (*MAT_ENHANED_COMPOSITE_DAMAGE) [58] on each lamina with suitable orientations in the subsequent macro-scale. The lamina model incorporates rate-dependence in strengths for tension, compression, and shear. The rate-dependent strengths are implemented as load curves in the LS-DYNA keyword file in conjunction with the composite damage material model. The lamina model coupled with interlaminar characteristics captures the experimentally observed deformed configurations with reasonable accuracy, as described in Section 3.2.3. The rate dependence of the tensile and compressive strengths is more pronounced than that of the shear strength. This trend is consistent with the shear behavior of the woven unit cell being primarily governed by the matrix behavior [73]. 


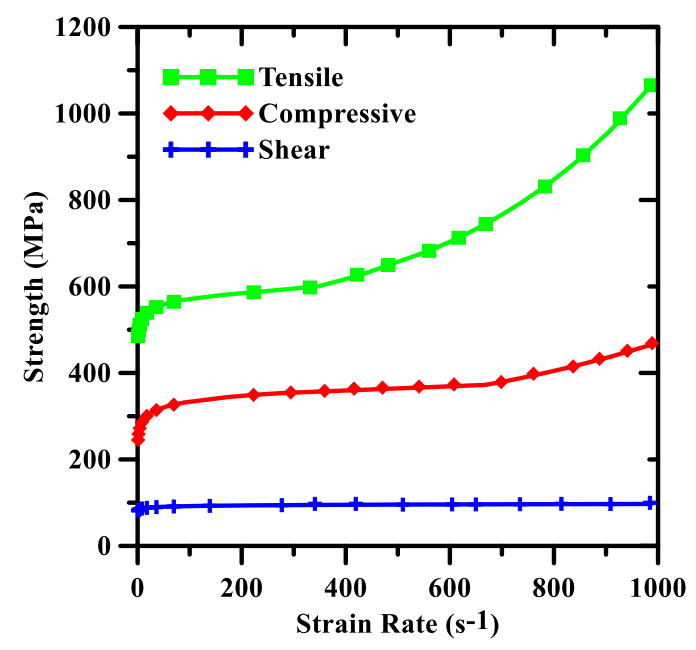

Figure 8. Rate-dependent homogenized strength properties for fabric lamina obtained from mesoscale simulations which serve as input to macro-scale cylinder for tension, compression, and shear

\subsubsection{Macro-scale Response and Correlation with Experimental Observations}

As described in the previous section, the rate-dependent composite responses obtained from mesoscale simulations (see Figure 8) serve as input to the macro-scale cylinder model. The rate-dependent load curves for the coating materials (polyurea and rubber) are adopted from the literature [7,16] and [74-77] respectively and are provided in Section B-3 of the Supplementary material. The interlaminar strengths are $35 \mathrm{MPa}$ and $68 \mathrm{MPa}$ along normal and shear directions respectively [16,72]. The critical energy release rates are $1.21 \mathrm{~N} / \mathrm{mm}$ and $4.55 \mathrm{~N} / \mathrm{mm}$ for normal and shear directions respectively [72]. The adopted interlaminar material properties are provided in Section B-3 of the Supplementary material. In this paper, the dimensions and ply orientations of the cylinder are kept the same as the ones adopted in the experiment. The coating thickness, coating material type, charge standoff distance, and the explosive charge are varied to generate the large dataset. In this section, a representative combination of material as well as the geometrical features, same as the one adopted in the experimental study, is presented for the ease of elucidation.

For the Eulerian components (see Section 3.1.4.2), RP-503 explosive, air, water the material properties, and EOS are adopted from the literature [8,16]. A nominal mesh size of $2.5 \mathrm{~mm}$ was adopted for the Lagrangian components [16] while the Euler mesh was implemented with elements of a nominal size of $25 \mathrm{~mm}$, similar to the mesh sizes adopted in a previous article [17]. The database keywords in LS-DYNA are used to obtain component-level properties (see Figure 9 for fluid density), pressure for virtual sensors (see Figure 10(a)), and component-level energies (see Figure 12).

The model is correlated with experimental observations before generating large datasets toward ML (see Section 4). Toward that end, the UNDEX behavior is captured in the wavefronts generated in the water 
domain. The near field explosions in the context of underwater structures are determined based on observation of spherical (non-uniform) incident wave on the structure. Since the stand-off distance to achieve near field condition varies based on explosive energy (mass and type of the explosive) and the geometrical dimensions of the structure, no particular cut-off for stand-off distance was chosen. Instead, the applicability of the near field explosion for generation of each data point in the dataset was carefully ensured by confirming the presence of spherical incident waves on the structure, as evidenced in Figure 9 for the simulated underwater explosion. Similar spherical wavefronts were observed in a previous study for nearfield underwater explosions [17]. Figures 9 (a) and (b) correspond to times of $0.0175 \mathrm{~ms}$ and $0.025 \mathrm{~ms}$ respectively.

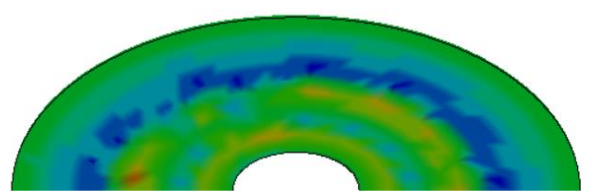

(a) $\begin{aligned} & \dot{0} \\ & \dot{0}\end{aligned}$

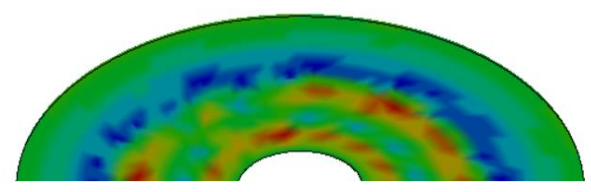

(b)

Figure 9. Sectional view of fluid domain showing wavefront at (a) $0.0175 \mathrm{~ms}$ and (b) $0.025 \mathrm{~ms}$ (Fringe levels indicate fluid density)

The pressure-time history for an RP-503 charge placed at a standoff distance of $5.08 \mathrm{~cm}$ is shown in Figure 10(a). The figure compares the experimental and simulated pressure-time histories. As observed from the figure, the simulated pressure-history shows a good correlation with experimental observation which establishes the efficacy of the multiscale simulation approach presented here. During the decay phase in Figure 10(a), the mismatch between the simulated and experimental pressure-time history can be explained from the fact that the simulated pressure history is obtained from an LS-Dyna keyword output, DBFSI [58] which averages the pressure in the water domain whereas, in the experiment, the pressure sensor records the pressure-time history at a particular point in space in the fluid domain. 

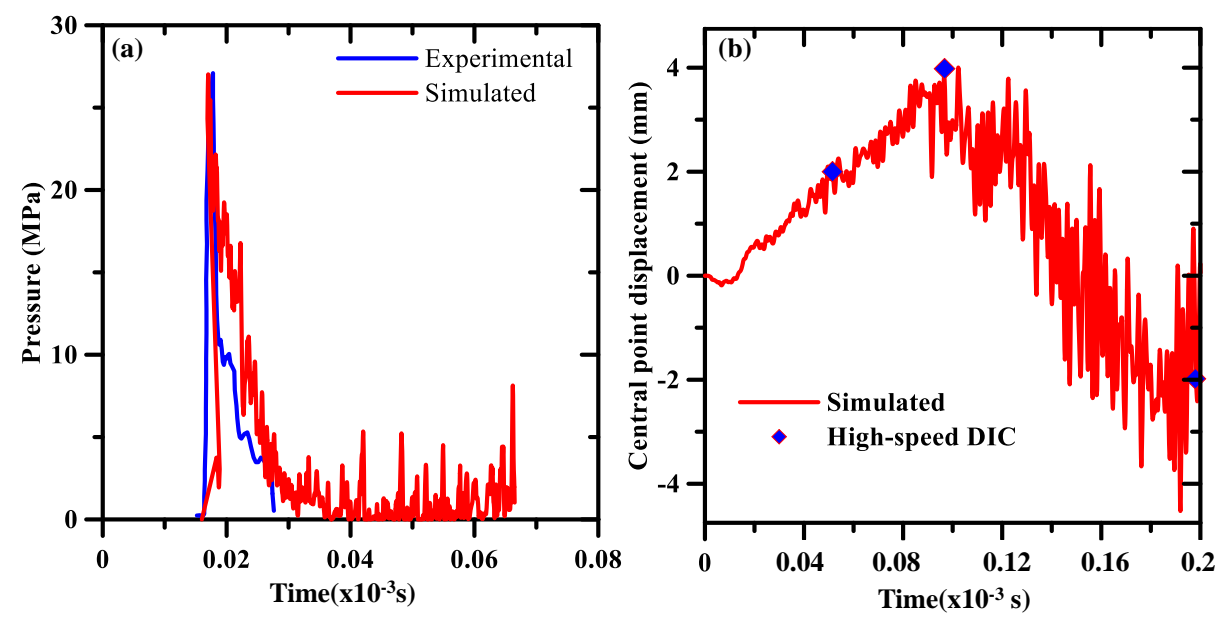

Figure 10. Correlation between experimental and simulated (a) pressure-time history (b) out-of-plane displacement for central point on loading face of cylinder for RP-503 charge at a stand-off distance of $5.08 \mathrm{~cm}$

Figure 10 (b) shows the simulated central point out-of-plane displacement-time history on the loading face of the cylinder which shows an excellent correlation with experimental values obtained from DIC. Please refer to Section $\mathrm{E}$ of the supplementary material for more details on the comparison between the experimental DIC data and the simulated responses.

Having correlated the pressure-history and cylinder deflections with experimental observations, the transient response of the coated cylinder configuration is studied. Owing to the implementation of element deletion in the model, the damage zones can be correlated with experimental observations. Figures 11 (ab) show the correlation between the damaged cylinder as observed from experiments and simulation. The effective plastic strain (a scalar variable that is calculated from the plastic component of the rate of deformation tensor and grows when the material yields [58]) on the damaged configuration is plotted in Figure $11(\mathrm{c})$. 


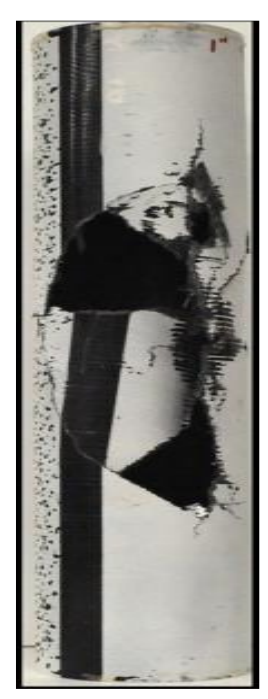

(a)

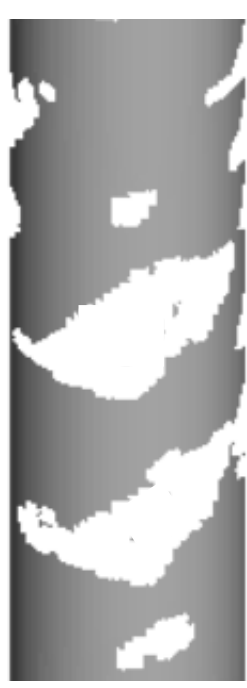

(b)

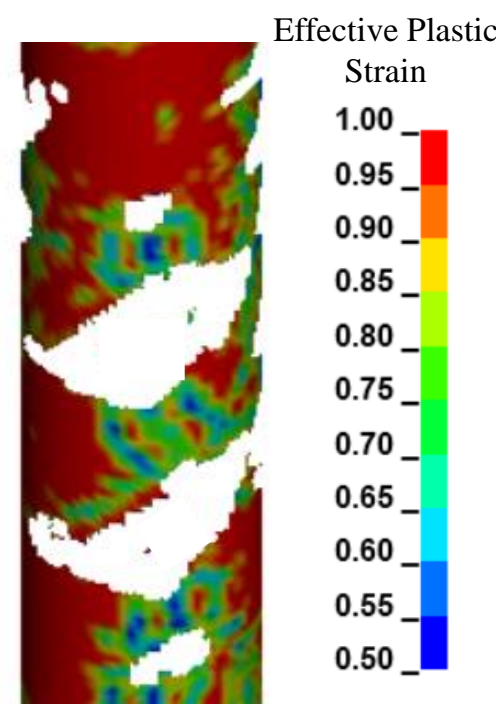

(c)

Figure 11. Uncoated configuration for RP-503 charge placed at $2.54 \mathrm{~cm}$ standoff showing: (a) experimental and (b) simulated damaged cylinder post-UNDEX; (c) simulated effective plastic strain (monotonically increasing scalar value that is a time integral of the plastic component of the rate of deformation tensor) contours on the damaged configuration

The energy-time histories from the time of the explosion are provided in Figure 12. The transient response of the coating and the cylinder are captured in Figure 12. While Figure 12 (a) shows the state of kinetic (KE) and internal energies (IE) in the cylinder and coating, Figure 12 (b) shows the conversion of the kinetic to internal energy. The energy transfer mechanism of the coated configuration can be ascertained from the time history. It is to be noted that at the initial stage, the peak of the KE/IE ratio indicates that the configuration obtains an instantaneous kinetic energy from elastic deformation, but the internal energy is minuscule. Just after the peak, the kinetic energy decreases while the internal energy increases until the ratio achieves a plateau indicating equal rates of growth of internal and kinetic energy or a stable state. The growth of internal energy as contour plots is shown in the inset pictures. 

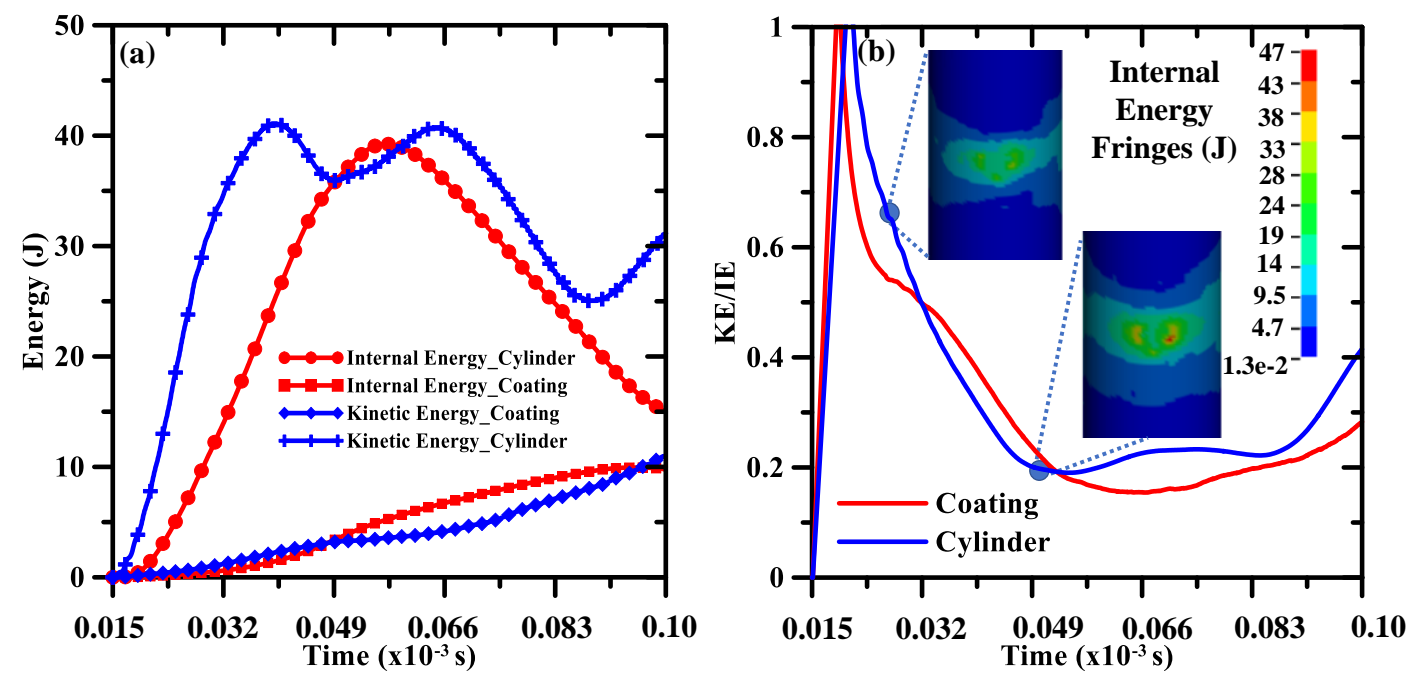

Figure 12. Component-level kinetic and internal energy for cylinder and coating for (a) Energy-time histories from time of explosion and (b) time history for the ratio of kinetic to internal energy (See insets with internal energy fringes) corresponding to an RP-503 charge placed at a stand-off distance of $5.08 \mathrm{~cm}$

\section{MACHINE LEARNING BASED PERFORMANCE PREDICTION}

\subsection{Machine Learning Approach}

Here, a feed-forward multilayer perceptron is used for training, and the weights are trained by backpropagation algorithm [19]. The technique of feedforward backpropagation multilayer perceptron is commonly referred to as neural network. The neural network is a mathematical model that enables mapping of a given set of predictors $x$ to a set of desired responses $y$ with the mapping comprising of layers of perceptron and activation functions. This mapping technique is referred to as a feed-forward neural network which provides an estimate response as in Equation 36.

$$
y=f_{N}\left(A_{N}, \ldots f_{2}\left(A_{2}, f_{1}\left(A_{1}, x\right)\right) \ldots\right)
$$

Where $f_{N}$ is the activation function and $A_{i}(i=1$ to $N)$ is the transformation matrix that contains the weights between two layers of perceptron. A constrained optimization problem shown in Equation 37 is used to solve the mapping.

$$
\operatorname{argmin}_{A_{j}}\left\{f_{N}\left(A_{N}, \ldots f_{2}\left(A_{2}, f_{1}\left(A_{1}, x\right)\right) \ldots\right)+\lambda g\left(A_{j}\right)\right\}
$$

Where $\lambda$ is the regularization intensity constant and $g$ is a functional form of the weights to be regularized. The optimization problem is usually solved by stochastic gradient descent or backward propagation algorithm. However, the solution to this optimization problem is not unique owing to the non-convex nature 
of the neural network. The selection of the number of layers and the number of perceptrons in each layer plays a significant role in prediction efficiency which necessitates proper regularization when the neural network is implemented. In the current study, a rectified linear unit (ReLU) is used for training the network.

\subsection{Dataset Generation}

The incident impulse on the structure plays an integral part in the response of underwater structures subjected to shock loading. The incident impulse is dependent on the energy released by the explosive and its distance from the surface of the structure (or stand-off distance). The ability of a structure to withstand shock is determined by its ability to dissipate the energy. In the current scope of this study, the efficacy of rate-dependent coatings applied to composite laminates is studied with regards to its performance in underwater shocks on an explosion event. Thus, the parameters chosen for study are the energy of the explosive, the stand-off distance, the ratio of coating thickness to the wall thickness of the cylinder, and the density of the coated laminate. While this study implements explosive energy, stand-off distance, the ratio of coating to the wall thickness, and material density as the input for the ML-based predictive model, the influence of various additional input performance descriptors such as variations in the model geometry, the sensitivity of imparted impulse, peak deflections, the extent of damage to charge, etc. on the prediction efficacy and the robustness of the model can be evaluated in a future study. Epoxy and polyvinyl ester are chosen as matrix materials for the woven laminate; polyurea and rubber are chosen for the coating. Although epoxy forms the obvious choice as a reference material, vinyl ester is chosen in this paper considering its effectiveness in marine structures. For the protective envelopes, both polyurea and rubber are chosen, owing to their plasticity and kinematic hardening at high strain rates leading to superior energy absorption. For dataset preparation, the material choice directly affects the mass of the system and in turn its density, the impact of which is discussed later. Additionally, the ratio of the coating thickness to the substrate thickness helps to capture the efficiency of the protective envelope. Figure 13 presents the contour of the peak internal energy of the coated cylinder subjected to a UNDEX event in a ternary plot showing the combinations of energy of explosive, stand-off distance, and coating thickness. The explosive energy data, obtained from a previous article [78] ranges from 0.70 to $1.56 \mathrm{MJ} / \mathrm{kg}$. The standoff distance ranges from 0 (implying surface detonation) to $25 \mathrm{~cm}$. The ratio of coating thickness to wall thickness is varied from 0.25 to 3.5 (with a fixed wall thickness of $1.14 \mathrm{~mm}$ for $0 / 45 / 45 / 0$ laminate). A total of 3837 combinations are generated for a large enough dataset toward ML training, testing, and cross-validation.

The adequacy of the dataset is exemplified by the model's performance (as shown later) besides the physicsbased simulations that help to capture the complex UNDEX FSI event. The balance of the dataset is ensured by varying the parameters in a uniform fashion so as to capture data from a sufficient number of admissible combinations. The dataset (during NN implementation) is split into training (80\%) and test (20\%) datasets. 
A similar split is followed during every fold in cross-validation of the training dataset during hyperparameter optimization using a fivefold cross-validation. A consistency of the data is maintained by the underlying FE simulations with adequate convergence. These techniques ensure the adequacy of the dataset that ensures that the data is balanced, representative, complete, and consistent. The ternary plot (Figure 13) along its axes runs from 0 to $100 \%$ of the range of the parameters. Here the percentages of the parameters are calculated by dividing the value of the parameter with its maximum value. For example, an explosive energy of $1.56 \mathrm{MJ} / \mathrm{kg}$ is assigned here as $100 \%$. It is to be noted that zero or null values of the internal energy are observed on the contour plot for inadmissible combinations (for instance, null explosive energy). A judicious choice has been made by selection of data points from very high energy explosive concentrations where the protective envelopes are effective with sandwiched structures [6]. Similarly, a higher share of data points has been generated for stand-off distances simulating near field explosions $[8,16]$.

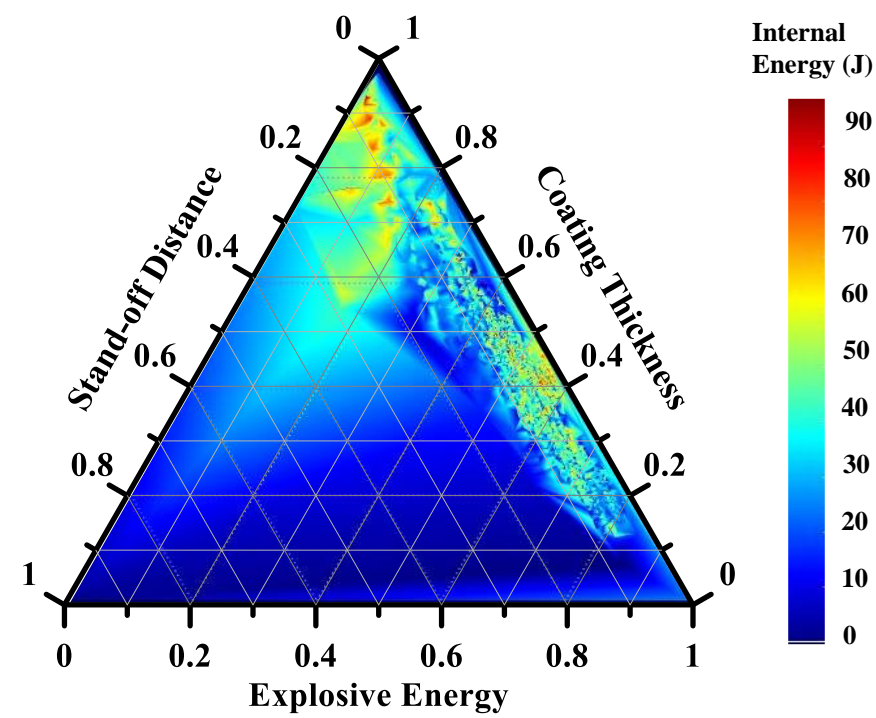

Figure 13. Contour plot of the internal energy of the coated laminate cylinder on a ternary plot of standoff distance, energy of explosive and coating thickness to represent the large dataset generated by parametric variation

The general trends of the correlation between the governing parameters can be ascertained from Figure 13. Since the mass of the structure governs the stored internal energy, with an increase in coating thickness, a higher internal energy is observed. On the other hand, the increase in explosive energy results in a higher overall energy in the system. The total explosive energy is transmitted as kinetic energy in the fluid and then translates to internal energy (see Figure 12(b)) of the coated laminate. A shorter standoff distance translates to a higher internal energy as observed in the peaks for low stand-off distances. The increasing 
trend continues till a threshold value is reached, beyond which it drops. A similar phenomenon is described in [79] for the nearfield underwater explosion response of structures.

In order to generate the large dataset, a python script implements message passing between the various scales. A flowchart is shown here in Figure 14 to elucidate the flow of information between the interactive length scales for dataset preparation. The data-generation scheme is implemented in Python which can invoke the respective solvers (ABAQUS ${ }^{\mathrm{TM}}$ and LS-Dyna). The main Python 3.7 program generates the scripts that can interface with both the solvers, ABAQUS ${ }^{\mathrm{TM}} /$ Explicit and LS-Dyna. This facilitates information transfer for the hierarchical multiscale framework. A brief description of the adopted methodology is provided hereafter.

The main program first generates an individual python script (.py) in the micro-scale that can be run in

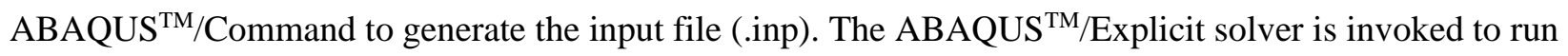
the input file and the output database (.odb) file is generated containing responses corresponding to different modes of loading (tension, compression, and shear). The main program then generates and implements another python script for post-processing of the .odb file. The homogenized response obtained from microscale analysis serves as input for mesoscale analysis which follows the same procedure as the one mentioned above for the microscale. Mesoscale responses serve as inputs for the macroscale simulations in LS-Dyna.

For LS-Dyna implementation, a keyword (.k) file is generated by the main program by using the effective mesoscale data which is thereafter run by invoking LS-Run so as to generate D3plots (.d3plot). An implicit check is triggered at the end of every iteration to ensure the consistency of information flow throughout the hierarchy and the information is logged. For double-precision Dyna solvers, open-source packages for automated post-processing are not yet available and it provides a scope for future work. In the current scope of this study, the peak energy is extracted from Dyna D3Plots.

Once the macroscale data is obtained and stored, the input parameters combination is continuously updated leading to micro-meso-macro simulations in a nested iterative process. The nested iterative process progresses for every material configuration as well as for different loading types in all the three length scales and thus generates a large dataset. The choice of Python as the platform for data generation facilitates the implementation of open-source packages scikit-learn, pandas, NumPy as essential toolboxes for ML, described in the subsequent sections. The modularity of the presented framework enables the re-utilization of modules for a variety of structural responses (viz. buckling of the cylinder) or coupled studies (viz. instability analysis with UNDEX). 


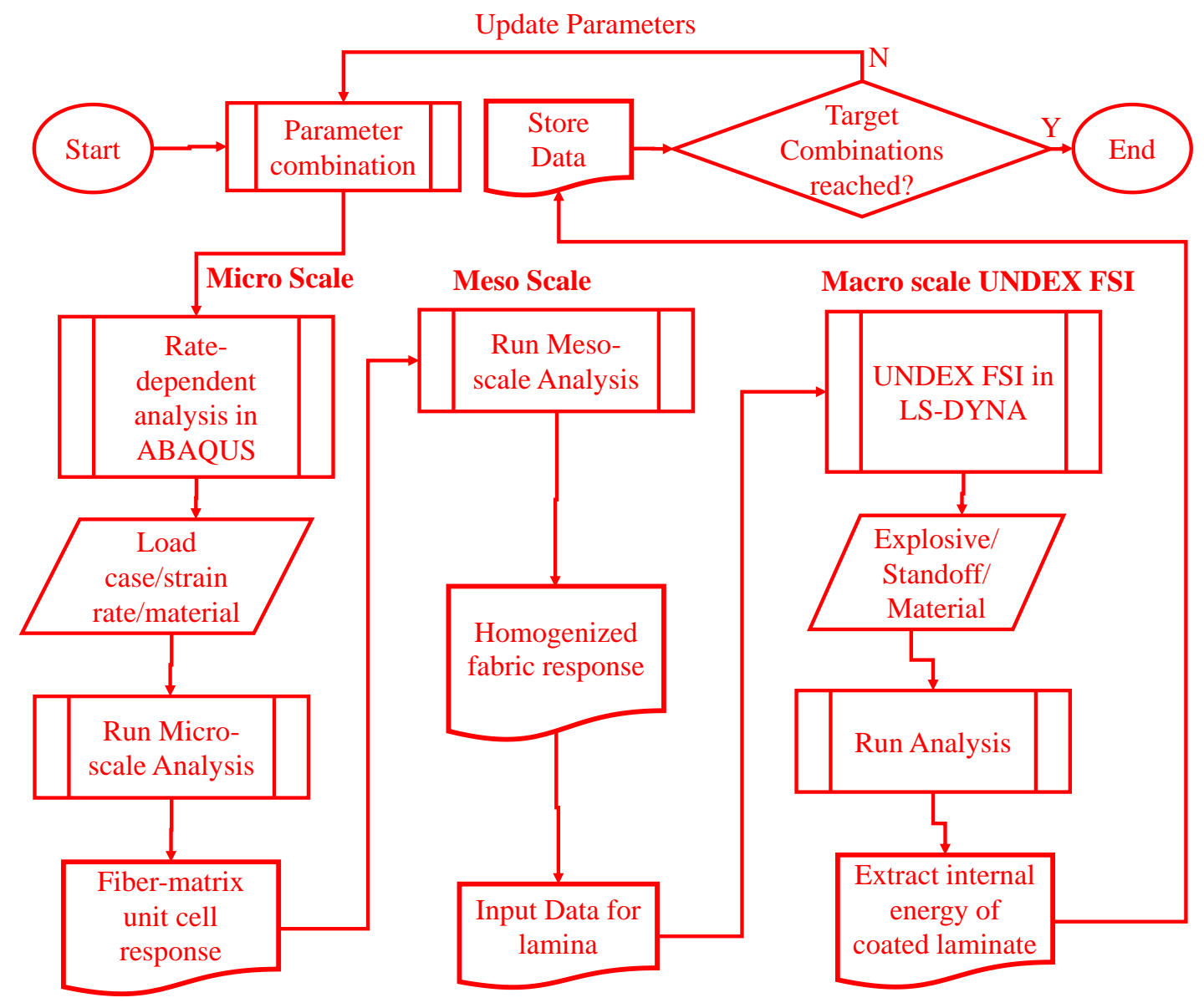

Figure 14. Data-generation scheme for multi-scale UNDEX simulation

\subsection{Machine Learning-Based Performance Prediction}

A multilayer perceptron (MLP) [19] which is a class of feedforward neural network containing an input layer, a hidden layer, and an output layer, is leveraged here. The MLP NN model is trained using the backpropagation algorithm [19]. Thus, hyperparameters such as the number of hidden layers, number of hidden nodes, optimizer, batch size, number of epochs need to be optimized prior to prediction. The loss function implemented for the back propagation in the neural network (NN) is mean squared error (MSE) loss; while for selecting the hyper-parameters such as number of neurons in NN, both MSE and $\mathrm{R}^{2}$ are used where a trade-off is sought for minimum MSE and high $\mathrm{R}^{2}$. The loss function is optimized using Adam Optimization Algorithm [80]. Figure 15 shows the MSE obtained from MLP-NN (with two hidden layers) with an increasing number of neurons. It is to be noted that the model complexity increases with the number of neurons. As observed from Figure 15, the MSE keeps on decreasing till 13 beyond which it achieves an inflection point and the MSE values start increasing. In other words, for an increasing number of neurons till 13, the model gets better at interpolating points. Thus, in this study, two hidden layers of thirteen hidden 
nodes are used to prevent overfitting of data. An Adam optimizer is adopted for the study with a learning rate of 0.001 and epochs at 400 for a batch size of 32. The optimized neural network is provided in Section F of the supplementary material.

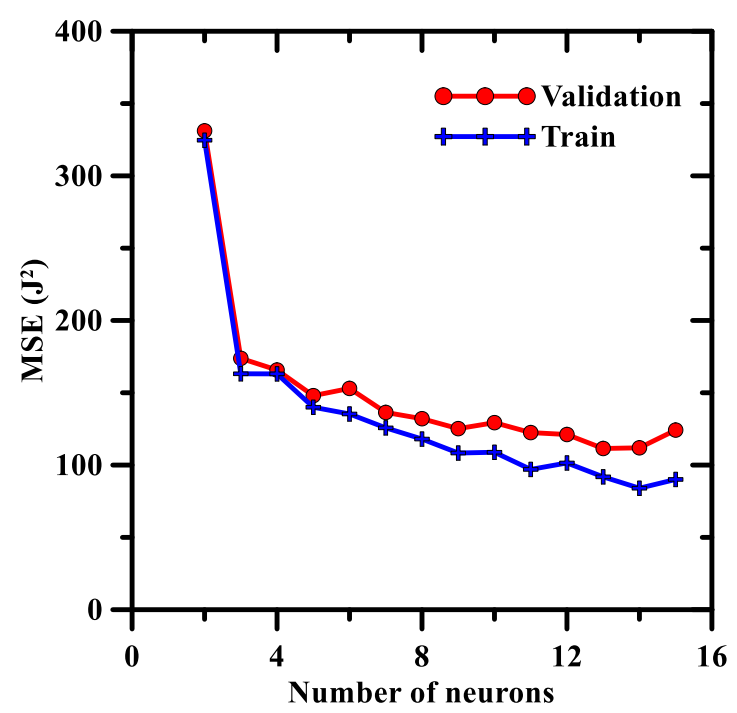

Figure 15. MSE values with varying number of neurons as obtained from training and validation data-sets (optimum number of neurons is chosen at minimum MSE) for peak internal energy

Figure 16 plots the predicted responses (using NN with 2 hidden layers and 13 neurons) against the measured values computed by multiscale FE simulations. Overall, a good prediction accuracy is achieved for the test dataset with an $\mathrm{R}^{2}$ value of 0.85 . This can be attributed to the robustness of the back-propagation method in NN-MLP.

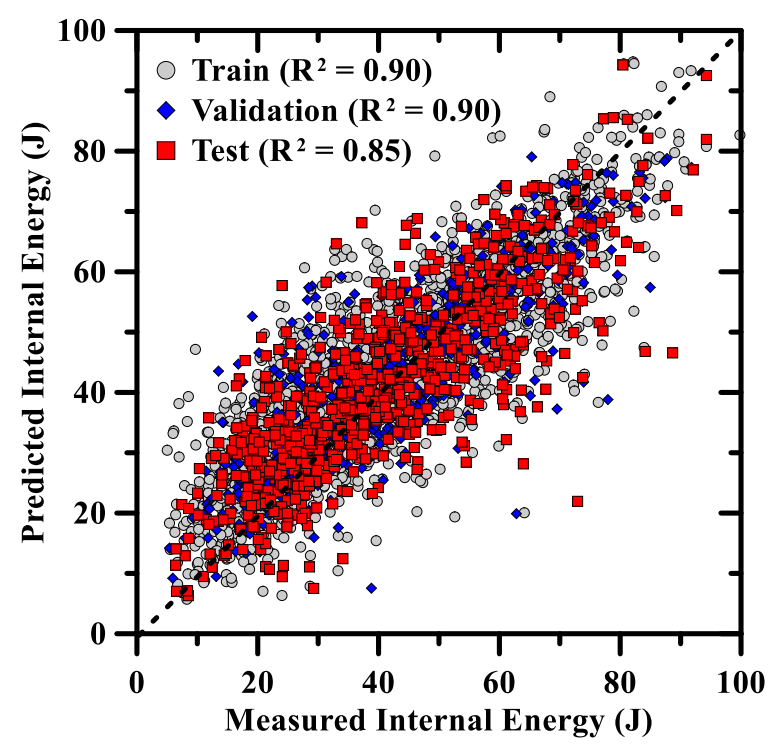


Figure 16. Comparison of predicted energies from NN (number of neurons equal to 13) and measured values from multiscale FSI simulations for large datasets of peak internal energy

The interpretability of the NN predictions using SHAP enables to ascertain the impact of each feature on the prediction. This is quantified by assigning an importance value to each feature toward a respective prediction. Figure 17 shows the SHAP values for each feature in the model (explosive energy, stand-off distance, ratio of coating to wall thickness, and material density).

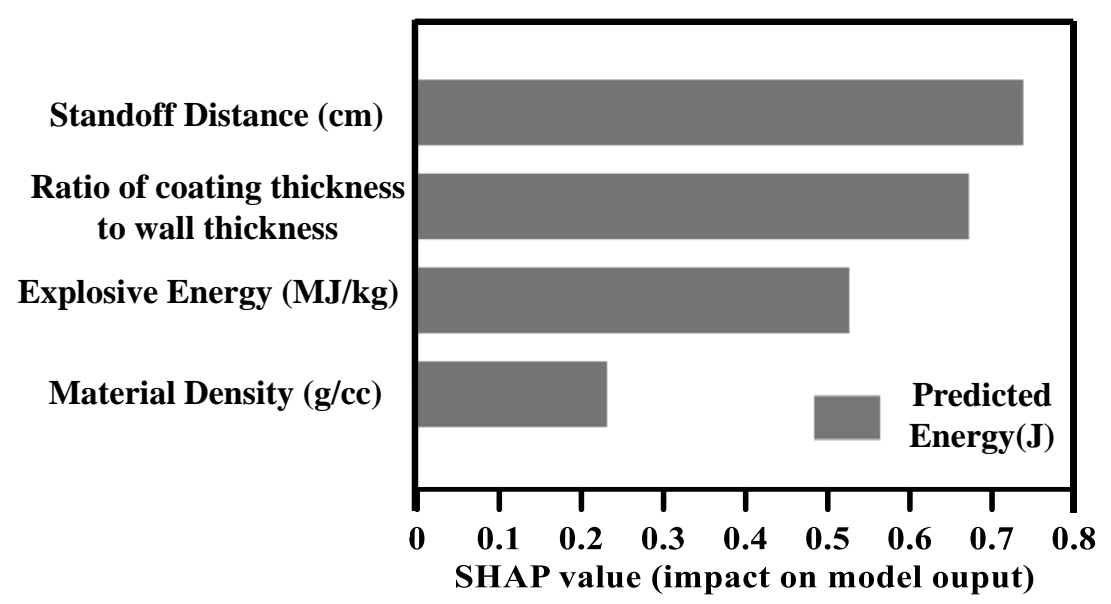

Figure 17. SHAP value for multiple features on target peak internal energy prediction of a laminated cylinder subjected to UNDEX

For the adopted range of the input parameters, standoff distance and the ratio of coating thickness to the wall thickness show a dominant effect on the model output followed by the explosive energy and the material density. Considering a fixed explosive energy-standoff distance combination, the ratio of coating thickness to the wall thickness shows a significantly higher influence on the model output as compared to density. Such results can have significant implications toward providing opportunities to tune the coating thickness for optimized energy dissipation in case of a nearfield underwater explosion event. While density plays a less significant role, it is to be noted that the density study in the current scope is limited to matrix materials or polymeric coatings and not foam type structures (with significantly stiff plates and very-lowdensity foams sandwiched in between) where a higher SHAP for material density would be anticipated.

\section{CONCLUSIONS}

This paper synergistically integrates high throughput FE simulations and ML to predict the dynamic response of coated composite cylinders subjected to the nearfield underwater explosion. Overall, the MLbased predictive technique showed excellent efficacy toward the prediction of the internal energy of the coated cylinder under nearfield explosion. The modular design of the simulation framework enables 
multiple components in a plug-and-play approach for modifications in either micro, meso, and macro scales. This provides the flexibility required for the incorporation of a plethora of responses that can result from imperfections introduced by manufacturing or environmental exposures.

In a bid to interpret the NN results and to elucidate the underlying governing features, a SHAP analysis is carried out. The results suggest that the stand-off distance and the energy of the explosive play a dominant role in the energy transmitted to the configuration whereby the mitigation effects are primarily pronounced by the coating thickness.

Overall, the performance prediction ability and the inferences from the study of coated laminated cylinders subjected to a near-field explosive event underscore the capability of the framework as a potential design optimizer. The key innovations brought about by the current scope of study are presented herewith: (a) a comprehensive multiscale strategy for effective property prediction of woven laminates for high-rate loadings under directional loading scenarios; (b) an encapsulated approach to effective property prediction and the subsequent utilization of such properties for explicit analysis of macro structures under complex high-rate loadings; (c) laying the foundation for an inheritance-based approach where effective material properties and or laminate characteristics can be obtained in a hybrid fashion using both experimental observations and the multiscale framework (for instance, the coating input properties are experimentally obtained whereas the laminate properties are simulated); (d) a polymorphic approach to studying the structural responses of coated laminated cylinders under explosive loadings where certain key material characteristics of the model can be re-used in a parametric environment by modulating variables such as explosive charge energy and stand-off distance (e)developing an open-source, user-extendable environment for relatively inexpensive computation of coating performance in a comparative framework by utilizing neural networks.

While the ML-based model presented here can be used as a starting point toward developing a user-friendly predictive tool for materials engineers and designers, the influence of some additional aspects such as (1) increase in the number of performance descriptors i.e., increase in the dimensionality of the input space, (2) using several neural network architectures such as changing the activation function at each hidden layer and the output layer, the size of neurons for each hidden layers, the weight initialization, normalizing or scaling data, different learning algorithm parameters, and (3) last but not the least increase in the number of data points within the dataset, on the predictive efficacy can be further investigated in a series of future studies toward building a robust and generalized tool. 


\section{DATA AVAILABILITY}

The raw data required to reproduce these findings are available to download from https://github.com/iamsumeru/Simulated-Dataset-UNDEX-Response-of-coated-composite-cylinders.git.

\section{Declaration of Competing Interest}

The authors declare that they have no known competing financial interests or personal relationships that could have appeared to influence the work reported in this paper.

\section{CRediT author statement}

Sumeru Nayak: Investigation, Data Curation, Software, Formal analysis, Visualization, Validation, Writing - Original Draft; Gideon A. Lyngdoh: Software, Formal analysis, Visualization, Validation, Writing Original Draft; Arun Shukla: Conceptualization, Resources, Writing - Review \& Editing, Funding acquisition; Sumanta Das: Conceptualization, Methodology, Resources, Writing - Review \& Editing, Supervision, Funding acquisition, Project administration.

\section{Acknowledgements}

The computational research was conducted in the Multiscale \& Multiphysics Mechanics of Materials Research Laboratory (M4RL) at the University of Rhode Island and the supports that have made this laboratory possible are acknowledged. The authors acknowledge Andromeda HPC and Bluewaves HighPerformance Research Computing at the University of Rhode Island for providing computer clusters and data storage resources that have contributed to the research results reported within this paper. The experiments were performed in the Dynamic Photomechanics Laboratory (DPML) at the University of Rhode Island. Arun Shukla would like to acknowledge the financial support provided by Dr. Y.D.S. Rajapakse at the Office of Naval Research under Grant No. N00014-20-1-2877.

\section{References}

[1] Mouritz AP, Gellert E, Burchill P, Challis K. Review of advanced composite structures for naval ships and submarines. Composite Structures 2001;53:21-42. https://doi.org/10.1016/S02638223(00)00175-6.

[2] Lu W. Novel protection mechanism of blast and impact waves by using nanoporous materials. Dynamic Behavior of Materials, Volume 1, Springer; 2016, p. 177-183.

[3] Fernando PLN, Mohotti D, Remennikov A. Behaviour of explosively welded impedance-graded multi-metal composite plates under near-field blast loads. International Journal of Mechanical Sciences 2019;163:105124. 
[4] Wanchoo P, Matos H, Rousseau C-E, Shukla A. Investigations on air and underwater blast mitigation in polymeric composite structures - A review. Composite Structures 2021;263:113530. https://doi.org/10.1016/j.compstruct.2020.113530.

[5] Abrate S. Coatings for Mitigating the Effects of Underwater Shock Waves on Structures. In: Gopalakrishnan S, Rajapakse Y, editors. Blast Mitigation Strategies in Marine Composite and Sandwich Structures, Singapore: Springer; 2018, p. 321-35. https://doi.org/10.1007/978-981-107170-6_17.

[6] Tekalur SA, Shukla A, Shivakumar K. Blast resistance of polyurea based layered composite materials. Composite Structures 2008;84:271-81. https://doi.org/10.1016/j.compstruct.2007.08.008.

[7] LeBlanc J, Shukla A. Response of polyurea-coated flat composite plates to underwater explosive loading. Journal of Composite Materials 2015;49:965-80. https://doi.org/10.1177/0021998314528263.

[8] LeBlanc J, Shillings C, Gauch E, Livolsi F, Shukla A. Near Field Underwater Explosion Response of Polyurea Coated Composite Plates. Exp Mech 2016;56:569-81. https://doi.org/10.1007/s11340-0150071-8.

[9] Li QM, Meng H. Attenuation or enhancement - a one-dimensional analysis on shock transmission in the solid phase of a cellular material. International Journal of Impact Engineering 2002;27:1049-65. https://doi.org/10.1016/S0734-743X(02)00016-7.

[10] Wadley HNG, Murty YV, Jones T, Gupta R, Burkins M. Hybrid periodic cellular material structures, systems, and methods for blast and ballistic protection. US9921037B2, 2018.

[11] Ball GJ, East RA. Shock and blast attenuation by aqueous foam barriers: influences of barrier geometry. Shock Waves 1999;9:37-47.

[12] Graham KJ, Sewell RGS. Blast attenuator. US4543872A, 1985.

[13] Hutchinson JW, Xue Z. Metal sandwich plates optimized for pressure impulses. International Journal of Mechanical Sciences 2005;47:545-69. https://doi.org/10.1016/j.ijmecsci.2004.10.012.

[14] Xue Z, Hutchinson JW. Preliminary assessment of sandwich plates subject to blast loads. Int J Mech Sci 2003:687-705.

[15] Wang E, Gardner N, Shukla A. The blast resistance of sandwich composites with stepwise graded cores. International Journal of Solids and Structures 2009;46:3492-502. https://doi.org/10.1016/j.ijsolstr.2009.06.004.

[16] Gauch E, LeBlanc J, Shukla A. Near field underwater explosion response of polyurea coated composite cylinders. Composite Structures 2018;202:836-52. https://doi.org/10.1016/j.compstruct.2018.04.048.

[17] Kim J-H, Shin H-C. Application of the ALE technique for underwater explosion analysis of a submarine liquefied oxygen tank. Ocean Engineering 2008;35:812-22. https://doi.org/10.1016/j.oceaneng.2008.01.019. 
[18] Gong SW. Transient response of stiffened composite submersible hull to underwater shock and bubble. Composite Structures 2019;213:243-51. https://doi.org/10.1016/j.compstruct.2019.01.097.

[19] Lyngdoh GA, Li H, Zaki M, Krishnan NMA, Das S. Elucidating the constitutive relationship of calcium-silicate-hydrate gel using high throughput reactive molecular simulations and machine learning. Scientific Reports 2020;10:21336. https://doi.org/10.1038/s41598-020-78368-1.

[20] Ramprasad R, Batra R, Pilania G, Mannodi-Kanakkithodi A, Kim C. Machine learning in materials informatics: recent applications and prospects. Npj Computational Materials 2017;3:1-13. https://doi.org/10.1038/s41524-017-0056-5.

[21] Lookman T, Alexander FJ, Rajan K. Information Science for Materials Discovery and Design. Springer Berlin; 2016.

[22] Curtarolo S, Setyawan W, Hart GLW, Jahnatek M, Chepulskii RV, Taylor RH, et al. AFLOW: An automatic framework for high-throughput materials discovery. Computational Materials Science 2012;58:218-26. https://doi.org/10.1016/j.commatsci.2012.02.005.

[23] Jain A, Ong SP, Hautier G, Chen W, Richards WD, Dacek S, et al. Commentary: The Materials Project: A materials genome approach to accelerating materials innovation. APL Materials 2013;1:011002. https://doi.org/10.1063/1.4812323.

[24] Anoop Krishnan NM, Mangalathu S, Smedskjaer MM, Tandia A, Burton H, Bauchy M. Predicting the dissolution kinetics of silicate glasses using machine learning. Journal of Non-Crystalline Solids 2018;487:37-45. https://doi.org/10.1016/j.jnoncrysol.2018.02.023.

[25] Cohen S, Ruppin E, Dror G. Cohen, S., Ruppin, E. \& Dror, G. Feature selection based on the Shapley value. Proceedings of the 19th international joint conference on Artificial intelligence, Morgan Kaufmann Publishers Inc; 2005.

[26] Lundberg SM, Lee S-I. A Unified Approach to Interpreting Model Predictions. Advances in Neural Information Processing Systems 2017;30:4765-74.

[27] Schreier H, Orteu J-J, Sutton MA. Image Correlation for Shape, Motion and Deformation Measurements. Boston, MA: Springer US; 2009. https://doi.org/10.1007/978-0-387-78747-3.

[28] Pandya KS, Veerraju Ch, Naik NK. Hybrid composites made of carbon and glass woven fabrics under $\begin{array}{lllll}\text { quasi-static loading. } & \text { Materials } & \text { D } & \text { Design }\end{array}$ https://doi.org/10.1016/j.matdes.2011.03.003.

[29] Nayak S, Das S. A microstructure-guided numerical approach to evaluate strain sensing and damage detection ability of random heterogeneous self-sensing structural materials. Computational Materials Science 2019;156:195-205.

[30] Nayak S, Das S. Strain sensing efficiency of hierarchical nano-engineered smart twill-weave composites: Evaluations using multiscale numerical simulations. Composite Structures 2021;255:112905. https://doi.org/10.1016/j.compstruct.2020.112905.

[31] Omairey SL, Dunning PD, Sriramula S. Development of an ABAQUS plugin tool for periodic RVE homogenisation. Engineering with Computers 2019;35:567-77. https://doi.org/10.1007/s00366-0180616-4. 
[32] Wang L, Wu J, Chen C, Zheng C, Li B, Joshi SC, et al. Progressive failure analysis of 2D woven composites at the meso-micro scale. Composite Structures 2017;178:395-405. https://doi.org/10.1016/j.compstruct.2017.07.023.

[33] Xia Z, Zhou C, Yong Q, Wang X. On selection of repeated unit cell model and application of unified periodic boundary conditions in micro-mechanical analysis of composites. International Journal of Solids and Structures 2006;43:266-78. https://doi.org/10.1016/j.ijsolstr.2005.03.055.

[34] Li S. Boundary conditions for unit cells from periodic microstructures and their implications. $\begin{array}{llll}\text { Composites Science } & \text { 2008;68:1962-74. }\end{array}$ https://doi.org/10.1016/j.compscitech.2007.03.035.

[35] Goldberg R, Roberts G, Gilat A. Implementation of an Associative Flow Rule Including Hydrostatic Stress Effects Into the High Strain Rate Deformation Analysis of Polymer Matrix Composites. Journal of Aerospace Engineering 2003;18. https://doi.org/10.1061/(ASCE)0893-1321(2005)18:1(18).

[36] Shokrieh MM, Mosalmani R, Omidi MJ. A strain-rate dependent micromechanical constitutive model for glass/epoxy composites. Composite Structures 2015;121:37-45. https://doi.org/10.1016/j.compstruct.2014.10.035.

[37] Salas PA, Benson DJ, Venkataraman S, Loikkanen MJ. Numerical Implementation of Polymer Viscoplastic Equations for High Strain-Rate Composite Models. Journal of Aerospace Engineering 2009;22:304-9. https://doi.org/10.1061/(ASCE)0893-1321(2009)22:3(304).

[38] Shams A, Panteghini A, Bardella L, Porfiri M. A micromechanical model to study failure of polymerglass syntactic foams at high strain rates. Computational Materials Science 2017;135:189-204. https://doi.org/10.1016/j.commatsci.2017.04.007.

[39] Li X, Binienda WK, Goldberg Robert K. Finite-Element Model for Failure Study of TwoDimensional Triaxially Braided Composite. Journal of Aerospace Engineering 2011;24.

[40] Jiang H, Ren Y, Zhang S, Liu Z, Nie L. Multi-scale finite element analysis for tension and ballistic penetration damage characterizations of 2D triaxially braided composite. J Mater Sci 2018;53:1007194. https://doi.org/10.1007/s10853-018-2248-x.

[41] Sharma A, Daggumati S, Gupta A, Van Paepegem W. On the prediction of the bi-axial failure envelope of a UD CFRP composite lamina using computational micromechanics: Effect of microscale parameters on macroscale stress-strain behavior. Composite Structures 2020;251:112605. https://doi.org/10.1016/j.compstruct.2020.112605.

[42] Catalanotti G, Furtado C, Scalici T, Pitarresi G, van der Meer FP, Camanho PP. The effect of throughthickness compressive stress on mode II interlaminar fracture toughness. Composite Structures 2017;182:153-63. https://doi.org/10.1016/j.compstruct.2017.09.014.

[43] Chevalier J, Camanho PP, Lani F, Pardoen T. Multi-scale characterization and modelling of the transverse compression response of unidirectional carbon fiber reinforced epoxy. Composite Structures 2019;209:160-76. https://doi.org/10.1016/j.compstruct.2018.10.076.

[44] Gao Z. Comprehensively simulating the mixed-mode progressive delamination in composite laminates. Purdue University, 2015. 
[45] Müller JM, Lopez NR, Klein EA, Hopmann C. Predicting the damage development in epoxy resins using an anisotropic damage model. Polymer Engineering \& Science 2020;60:1324-32. https://doi.org/10.1002/pen.25383.

[46] Zheng X. Nonlinear Strain Rate Dependent Composite Model for Explicit Finite Element Analysis. University of Akron, 2006.

[47] Ma D, Manes A, Amico SC, Giglio M. Ballistic strain-rate-dependent material modelling of glassfibre woven composite based on the prediction of a meso-heterogeneous approach. Composite Structures 2019;216:187-200. https://doi.org/10.1016/j.compstruct.2019.02.102.

[48] Xu L, Huang Y, Zhao C, Ha SK. Progressive failure prediction of woven fabric composites using a multi-scale approach. International Journal of Damage Mechanics 2018;27:97-119. https://doi.org/10.1177/1056789516663613.

[49] Scazzosi R, Manes A, Petrone G, Giglio M. Two different modelling approaches for fabric composites subjected to ballistic impact. IOP Conf Ser: Mater Sci Eng 2018;406:012051. https://doi.org/10.1088/1757-899X/406/1/012051.

[50] Zeng Q, Sun L, Ge J, Wu W, Liang J, Fang D. Damage characterization and numerical simulation of shear experiment of plain woven glass-fiber reinforced composites based on 3D geometric $\begin{array}{llll}\text { reconstruction. } & \text { Composite } & \text { 2020;233:111746. }\end{array}$ https://doi.org/10.1016/j.compstruct.2019.111746.

[51] Brown LP, Long AC. 8 - Modeling the geometry of textile reinforcements for composites: TexGen. In: Boisse P, editor. Composite Reinforcements for Optimum Performance (Second Edition), Woodhead Publishing; 2021, p. 237-65. https://doi.org/10.1016/B978-0-12-819005-0.00008-3.

[52] Louise Brown, mike-matveev, georgespackman. louisepb/TexGen: TexGen v3.12.0. Zenodo; 2020. https://doi.org/10.5281/zenodo.3706478.

[53] Yan S, Zeng X, Long A. Meso-scale modelling of 3D woven composite T-joints with weave variations. Composites Science and Technology 2019;171:171-9. https://doi.org/10.1016/j.compscitech.2018.12.024.

[54] Hale P, Ng E. Non-linear material characterization of CFRP with FEM utilizing cohesive surface considerations validated with effective tensile test fixturing. Materials Today Communications 2020;23:100872. https://doi.org/10.1016/j.mtcomm.2019.100872.

[55] Sharma A, Daggumati S. Computational micromechanical modeling of transverse tensile damage behavior in unidirectional glass fiber-reinforced plastic composite plies: Ductile versus brittle fracture mechanics approach. International Journal of Damage Mechanics 2020;29:943-64. https://doi.org/10.1177/1056789519894379.

[56] Zhi J, Zhao L, Zhang J, Liu Z. A Numerical Method for Simulating the Microscopic Damage Evolution in Composites Under Uniaxial Transverse Tension. Appl Compos Mater 2016;23:255-69. https://doi.org/10.1007/s10443-015-9459-y.

[57] Zhang J, Zhang X. Simulating low-velocity impact induced delamination in composites by a quasistatic load model with surface-based cohesive contact. Composite Structures 2015;125:51-7. https://doi.org/10.1016/j.compstruct.2015.01.050. 
[58] 1s-dyna_971_manual_k.pdf n.d.

[59] Zhou G, Sun Q, Li D, Meng Z, Peng Y, Chen Z, et al. Meso-scale modeling and damage analysis of carbon/epoxy woven fabric composite under in-plane tension and compression loadings. International Journal of Mechanical Sciences 2021;190:105980. https://doi.org/10.1016/j.ijmecsci.2020.105980.

[60] DS Support Knowledge Base n.d. https://support.3ds.com/knowledge-base/ (accessed December 31, 2020).

[61] Donea J, Huerta A, Ponthot J-P, Rodríguez-Ferran A. Arbitrary Lagrangian-Eulerian Methods. Encyclopedia of Computational Mechanics Second Edition, American Cancer Society; 2017, p. 123. https://doi.org/10.1002/9781119176817.ecm2009.

[62] Grubenmann M, Heingärtner J, Hora P, Bassan D. Influence of temperature on in-plane and out-ofplane mechanical behaviour of GFRP composite. J Phys: Conf Ser 2018;1063:012146. https://doi.org/10.1088/1742-6596/1063/1/012146.

[63] Ogihara S, Koyanagi J. Investigation of combined stress state failure criterion for glass fiber/epoxy interface by the cruciform specimen method. Composites Science and Technology 2010;70:143-50. https://doi.org/10.1016/j.compscitech.2009.10.002.

[64] Zhang H, Ericson ML, Varna J, Berglund LA. Transverse single-fibre test for interfacial debonding in composites: 1. Experimental observations. Composites Part A: Applied Science and Manufacturing 1997;28:309-15. https://doi.org/10.1016/S1359-835X(96)00123-6.

[65] Romanowicz M. A numerical approach for predicting the failure locus of fiber reinforced composites under combined transverse compression and axial tension. Computational Materials Science 2012;51:7-12. https://doi.org/10.1016/j.commatsci.2011.07.039.

[66] Romanowicz M. Determination of the first ply failure load for a cross ply laminate subjected to uniaxial tension through computational micromechanics. International Journal of Solids and Structures 2014;51:2549-56. https://doi.org/10.1016/j.ijsolstr.2014.03.030.

[67] Akash Sharma, , Subbareddy Daggumati. Computational micromechanical modeling of transverse tensile damage behavior in unidirectional glass fiber-reinforced plastic composite plies: Ductile versus brittle fracture mechanics approach -. International Journal of Damage Mechanics 2020.

[68] Alireza Khademi, Mahmood M Shokrieh, Shahram Etemad Haghighi. A novel model to predict the stiffness and strength of unidirectional glass/epoxy composites at different strain rates -. Journal of Composite Materials 2020.

[69] Torabizadeh MA. Tensile, compressive and shear properties of unidirectional glass/epoxy composites subjected to mechanical loading and low temperature services. IJEMS Vol20(4) [August 2013] 2013.

[70] Shokrieh MM, Omidi MJ. Tension behavior of unidirectional glass/epoxy composites under different strain rates. Composite $\quad$ Structures $2009 ; 88: 595-601$. https://doi.org/10.1016/j.compstruct.2008.06.012.

[71] Gama BA, Gillespie JW. Finite element modeling of impact, damage evolution and penetration of thick-section composites. International Journal of Impact Engineering 2011;38:181-97. https://doi.org/10.1016/j.ijimpeng.2010.11.001. 
[72] Schwab M, Pettermann HE. Modelling and simulation of damage and failure in large composite components subjected to impact loads. Composite Structures 2016;158:208-16. https://doi.org/10.1016/j.compstruct.2016.09.041.

[73] Foroutan R, Nemes J, Ghiasi H, Hubert P. Experimental investigation of high strain-rate behaviour of fabric composites. Composite Structures 2013;106:264-9. https://doi.org/10.1016/j.compstruct.2013.06.014.

[74] Kolling S, Bois PAD, Benson DJ, Feng WW. A tabulated formulation of hyperelasticity with rate effects and damage. Comput Mech 2007;40:885-99. https://doi.org/10.1007/s00466-006-0150-x.

[75] Kolling S. A Simplified Rubber Model with Damage. . LS 2005:10.

[76] Kumar D, Sarangi S. Data on the viscoelastic behavior of neoprene rubber. Data in Brief 2018;21. https://doi.org/10.1016/j.dib.2018.10.081.

[77] Trivedi AR, Siviour CR. A Simple Rate-Temperature Dependent Hyperelastic Model Applied to Neoprene Rubber. J Dynamic Behavior Mater 2020;6:336-47. https://doi.org/10.1007/s40870-02000252-w.

[78] Keshavarz MH, Bagheri V. A Simple Correlation for Assessment of the Shock Wave Energy in Underwater Detonation. ZAAC 2019;645:1146-52.

[79] He Z, Chen Z, Jiang Y, Cao X, Zhao T, Li Y. Effects of the standoff distance on hull structure damage subjected to near-field underwater explosion. Marine Structures 2020;74:102839. https://doi.org/10.1016/j.marstruc.2020.102839.

[80] Kingma DP, Ba J. Adam: A Method for Stochastic Optimization. ArXiv:14126980 [Cs] 2017. 